\title{
Average values of some Z-parameters in a random set partition
}

\author{
Anisse Kasraoui* \\ Fakultät für Mathematik, Universität Wien \\ Nordbergstraße 15, A-1090 Vienna, Austria \\ anisse.kasraoui@gmail.com
}

Submitted: April 6, 2011; Accepted: Nov 21, 2011; Published: Dec 5, 2011

Mathematics Subject Classification: 05A18; 05A15, 05A16

\begin{abstract}
We find exact and asymptotic formulas for the average values of several statistics on set partitions: of Carlitz's $q$-Stirling distributions, of the numbers of crossings in linear and circular representations of set partitions, of the numbers of overlappings and embracings, and of the numbers of occurrences of a 2-pattern.
\end{abstract}

\section{Introduction}

Among all basic combinatorial structures, set partitions are probably one of the most attractive. Like their close cousin, the permutations, they have a remarkably rich combinatorial structure. This is partly due to the fact that they can be represented in many equivalent way: in terms of words, functions, graphs, placements of non-attacking rooks in a triangular diagram, line diagrams, etc. (see Figure 1 for some examples). During the past decades several statistics (here, statistic means an integer valued combinatorial parameter), also called parameters, on these different representations of set partitions have been introduced and studied. For instance, natural statistics on set partitions are the number of inversions in restricted growth functions or the numbers of crossings of arcs in linear and circular representations of set partitions. In very rare cases, one can determine the exact distribution of the statistic (i.e., for each $n$, the number of set partitions of $\{1,2, \ldots, n\}$ which have a certain value of the considered statistic), but most often, even though one has good information on the parameter, such as, for example, in form of an explicit formula for the generating function, there is no exact expression for the

${ }^{*}$ The author was supported by grant no. 090038012 from the Icelandic Research Fund and grant S9607-N13 from Austrian Science Foundation FWF in the framework of the National Research Network "Analytic Combinatorics and Probabilistic Number Theory". 


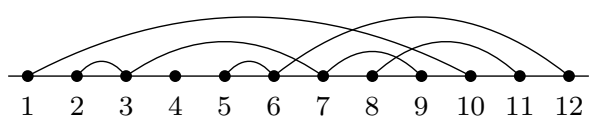

(a) linear representation

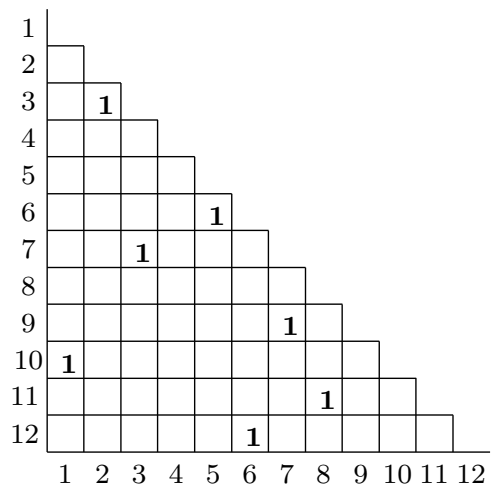

(c) rook placement

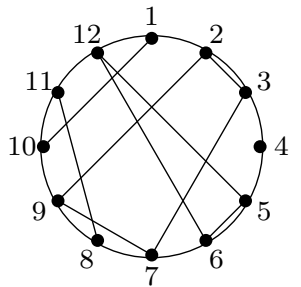

(b) circular representation

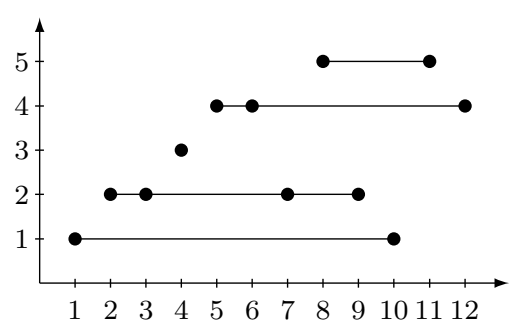

(d) line diagram

Figure 1: Some representations of the set partition $\pi=110 / 2379 / 4 / 5612 / 811$

distribution. The by far most important descriptive quantity associated to a statistic is its average value (also called mean value or expectation). The purpose of this paper is to compute the average values of several statistics on set partitions. Some of these statistics have been introduced in the literature previously and others are new.

\section{$2 \quad$ Background and main results}

\subsection{Basic facts}

The central objects of this paper are set partitions. A partition $\pi$ of a set $S$ is a collection of nonempty and mutually disjoint sets $\pi=\left\{A_{1}, A_{2}, \ldots, A_{k}\right\}$ whose union is $S$. The sets $A_{i}$ are called the blocks of the partition, and a partition into $k$ blocks is called a $k$-partition. Let us denote by $\Pi(S)$ the family of all partitions of $S$ and by $\Pi^{k}(S)$ the family of all $k$-partitions of $S$. For instance, $\Pi^{2}(\{a, b, c, d\})$ consists of the elements:

$$
\begin{array}{llll}
\{\{a\},\{b, c, d\}\} & \{\{b\},\{a, c, d\}\} & \{\{c\},\{a, b, d\}\} & \{\{d\},\{a, b, c\}\} \\
\{\{a, b\},\{c, d\}\} & \{\{a, c\},\{b, d\}\} & \{\{a, d\},\{b, c\}\} &
\end{array}
$$

The number $S_{n, k}$ of $k$-partitions of an $n$-set is called Stirling number of the second kind, while the number $B_{n}$ of all partitions of an $n$-set is called Bell number. Hence, by definition, we have $B_{n}=\sum_{k=1}^{n} S_{n, k}$. 
Almost all statistics considered on set partitions require that the set $S$ to be partitioned is linearly ordered. Without loss of generality, from now on, we will consider only partitions of subsets of the set $\mathbb{P}=\{1,2, \ldots\}$ of positive integers. With $[n]:=\{1,2, \ldots, n\}$, we will set $\Pi_{n}:=\Pi([n]), \Pi_{n}^{k}:=\Pi^{k}([n])$ and $\Pi:=\bigcup_{n \geq 1} \Pi_{n}$. A convenient way, called standard form (also called canonical order), to write a set partition $\pi \in \Pi(S), S \subseteq \mathbb{P}$, is to arrange the blocks of $\pi$ in increasing order of their minima and each block in increasing order. For instance, the partition $\pi$ of [9] consisting of the five blocks $\{1,4,7\},\{3,9\},\{2\},\{5\}$ and $\{6,8\}$ is written in standard form as $\pi=147 / 2 / 39 / 5 / 68$.

A substantial number of combinatorial statistics on set partitions were defined in terms of the restricted growth function representation for set partitions. It is well-known that $k ! S_{n, k}$ is the number of surjections from an $n$-set to a $k$-set. Actually, Stirling numbers also count a class of functions, called restricted growth functions. A word (or equivalently, a mapping) $w=w_{1} w_{2} \cdots w_{n}$ is said to be a restricted growth function (RGF for short) of length $n$ and size $k$ if:

1. $w_{1}=1$,

2. $w_{i+1} \leq \max \left\{w_{1}, w_{2}, \ldots, w_{i}\right\}+1$ for $1 \leq i \leq n-1$,

3. $\max \left\{w_{1}, w_{2}, \ldots, w_{n}\right\}=k$.

The usual way to associate bijectively set partitions of $[n]$ into $k$ blocks and RGF of length $n$ and size $k$ works as follows: to a partition $\pi=B_{1} / B_{2} / \cdots / B_{k}$ of $[n]$ written in standard form, we associate the RGF $w(\pi)=w_{1} w_{2} \ldots w_{n}$ such that $w_{i}=j$ if and only if $i \in B_{j}$. For instance, under this correspondence, the partition $\pi=147 / 2 / 39 / 5 / 68$ is sent to the RGF $w(\pi)=123145153$. The reader who knows the plethora of permutation and word statistics can imagine the amount of statistics that can be defined on set partitions via restricted growth functions. For instance, several statistics were defined in terms of inversions and noninversions, and more generally, in terms of counting occurrences of patterns in restricted growth functions.

Other interesting representations of set partitions suggest other combinatorial parameters. We will present some of them when they are needed.

\subsection{Z-statistics and main results}

Computation of average values of combinatorial parameters is often entertaining, but can be a very difficult task in some cases (for instance, consider the parameter "length of the longest increasing subsequence" in permutations). In this paper, we will consider some statistics whose computation of their (at least exact) average value is not trivial, but not as difficult as the one we mentioned previously. For instance, two of the parameters considered in the present paper are the numbers of crossings in linear and circular representations of set partitions (see Figure 1). A little moment's thought will convince 
the reader that the computation of their (at least exact) average value is not completely trivial.

Depending on which statistic on set partitions is considered, it is more or less difficult to find its average value. As an example, let us consider the statistics 'number of blocks' and 'number of crossings'. While a simple direct combinatorial argument gives the average number of blocks in a random set partition of $[n]$ (it is equal to $\frac{B_{n+1}}{B_{n}}-1$ ), a moment's thought will convince the reader that the computation of the average number of crossings in the circular representation of a random set partition of $[n]$, which, as we shall see later, is actually equal to

$$
\frac{n}{2} \frac{B_{n+1}}{B_{n}}+\frac{3 n}{2}-\frac{n(4 n+1)}{2} \frac{B_{n-1}}{B_{n}}-\left(\begin{array}{l}
n \\
2
\end{array}\right) \frac{B_{n-2}}{B_{n}}+\left(\begin{array}{l}
n \\
4
\end{array}\right) \frac{B_{n-4}}{B_{n}}
$$

is not completely trivial.

Perhaps the most natural and powerful approach to deal with average values is to use the linearity of the expectation by finding "nice decompositions" of the statistics we consider. This was done in the author's thesis [20] for the average number of crossings in the linear representation of a random set partition. It turns out that the decomposition used there is shared by a large number of existing statistics on set partitions and that their average values can be obtained almost "mechanically". In order to make these remarks more precise, we need additional terminology.

Given a subset $S \subseteq \mathbb{P}$ with cardinality $|S|=n$, the standardization map $\mathrm{st}_{S}$ is the (unique) order-preserving bijection $s_{S}: S \rightarrow[n]$. When the subset $S$ is clear from the context, we will drop the subscript. We let st $t_{S}$ act element-wise on objects built using $S$ as label. For instance, the set partition $\pi=29 / 410 / 5 / 711 / 8$ of $S=\{2,4,5,7,8,9,10,11\}$ is sent after standardization to the set partition $s t(\pi)=16 / 27 / 3 / 48 / 5$.

Definition 2.1. A set partition statistic STAT is said to be a Z-statistic if for any $\pi \in \Pi$,

$$
\operatorname{STAT}(\pi)=\sum_{\substack{A, B \in \pi \\ A \neq B}} \operatorname{STAT}(\operatorname{st}(\{A, B\})) .
$$

The terminology is inspired from a paper of Galovich and White [14], but originally, the term $Z$-statistic is for a famous statistic introduced by Bressoud and Zeilberger [2]. It is worth noting that via the RGF representation, our Z-statistics on set partitions could be seen as $Z$-statistics on words (with the definition of Galovitch and White).

For the present paper, the interest of Z-statistics on set partitions is that the computation of their average value in a random partition is essentially equivalent to the computation of their average value in a random 2-partition (see Theorem 3.1), the latter being often easier to calculate.

An important number of existing statistics on set partitions are in fact Z-statistics. In this paper, we will compute average values of Carlitz's $q$-Stirling distributions, the numbers of crossings in linear and circular representations, overlappings and embracings, and the number of occurrences of a 2-pattern in restricted growth functions. 


\subsubsection{Carlitz's $q$-Stirling distributions}

The most common $q$-analogs of Stirling numbers of the second kind found in the literature are probably the $q$-Stirling numbers $S_{n, k}(q)$ and $\tilde{S}_{n, k}(q)$ essentially described by Carlitz and Gould $[3,16]$. They can be defined as follows:

$$
S_{n, k}(q)=q^{k-1} S_{n-1, k-1}(q)+[k]_{q} S_{n-1, k}(q) \quad(n \geq k \geq 1),
$$

and $\tilde{S}_{n, k}(q)=q^{-\left(\begin{array}{c}k \\ 2\end{array}\right)} S_{n, k}(q)$, with $S_{n, k}(q)=\tilde{S}_{n, k}(q)=\delta_{n k}$ if $n=0$ or $k=0$.

Our interest in these $q$-Stirling numbers comes from the fact that they arise as generating functions for the distribution of several statistics on set partitions. Such statistics have been given by Milne [27], Garsia and Remmel [15], Sagan [30], Wachs and White [34], White [35], Leroux [25], de Médicis and Leroux [7], Ehrenborg and Readdy [9], Ksavrelof and Zeng [24]. This list is probably not exhaustive. It is natural to ask for the expectation of these " $q$-Stirling distributions".

We will say that a parameter stat defined on set partitions has the $q$-Stirling distribution $S(q)\left(\tilde{S}(q)\right.$, respectively) if its generating function on each $\Pi_{n}^{k}$ is given by $S_{n, k}(q)$ $\left(\tilde{S}_{n, k}(q)\right.$, respectively), i.e., for any $n \geq k \geq 1$, we have

$$
\sum_{\pi \in \Pi_{n}^{k}} q^{\text {stat }(\pi)}=S_{n, k}(q)\left(=\tilde{S}_{n, k}(q), \text { respectively }\right) .
$$

Theorem 2.2. Let stat be a parameter with the q-Stirling distribution $S(q)$. Then the average value $\mu_{n}$ of stat in a random set partition of $[n]$ satisfies

$$
\begin{aligned}
\mu_{n} & =-\frac{1}{4} \frac{B_{n+2}}{B_{n}}+\left(\frac{n}{2}+\frac{1}{4}\right) \frac{B_{n+1}}{B_{n}}+\left(-\frac{n}{2}+\frac{1}{4}\right) \\
& =\frac{n^{2}}{2 \log n}\left(1+\frac{\log \log n}{\log n}(1+o(1))\right) \quad \text { as } n \rightarrow \infty .
\end{aligned}
$$

The average value $\mu_{n, k}$ of stat in a random set partition of $[n]$ into $k$ blocks satisfies

$$
\begin{aligned}
\mu_{n, k} & =\frac{1}{2} n(k-1)-\frac{1}{2}\left(\begin{array}{l}
k \\
2
\end{array}\right)+\frac{1}{2}(n+1-k) \frac{S_{n, k-1}}{S_{n, k}} \\
& =\frac{1}{2} n(k-1)-\frac{1}{2}\left(\begin{array}{l}
k \\
2
\end{array}\right)+o(1) \quad \text { as } n \rightarrow \infty .
\end{aligned}
$$

Note that it is almost immediate to derive from the above results the expectations of the $q$-Stirling distribution $\tilde{S}(q)$, which we denoted by $\tilde{\mu}_{n}$ and $\tilde{\mu}_{n, k}$, respectively. Indeed, by the relation $\tilde{S}_{n, k}(q)=q^{-\left(\begin{array}{c}k \\ 2\end{array}\right)} S_{n, k}(q)$, we have

$$
\tilde{\mu}_{n, k}=\mu_{n, k}-\left(\begin{array}{l}
k \\
2
\end{array}\right) \quad \text { and } \quad \tilde{\mu}_{n}=\mu_{n}-\frac{1}{B_{n}} \sum_{k=1}^{n}\left(\begin{array}{l}
k \\
2
\end{array}\right) S_{n, k},
$$


and thus

$$
\begin{aligned}
\tilde{\mu}_{n, k} & =\frac{1}{2} n(k-1)-\frac{3}{2}\left(\begin{array}{l}
k \\
2
\end{array}\right)+\frac{1}{2}(n+1-k) \frac{S_{n, k-1}}{S_{n, k}} \\
\tilde{\mu}_{n} & =-\frac{3}{4} \frac{B_{n+2}}{B_{n}}+\left(\frac{n}{2}+\frac{7}{4}\right) \frac{B_{n+1}}{B_{n}}-\left(\frac{n}{2}+\frac{1}{4}\right),
\end{aligned}
$$

where the last equality follows from the identity $\sum_{k=1}^{n} k(k-1) S_{n, k}=B_{n+2}-3 B_{n+1}+B_{n}$ (for a proof, see e.g. [29]).

Almost all known statistics with the $q$-Stirling distribution $S(q)$ are in fact Z-statistics. In order to prove Theorem 2.2, we will work with the first known parameter with the distribution $S(q)$ : this is the statistic los (originally denoted $I^{m}$ ), first introduced by Milne (see [27, Section 4]) to answer a question of Garsia. The parameter los can also be defined by using the non-attacking rook placement model for set partitions (see e.g. [34]).

It is not hard to see that los can be defined for a set partition $\pi=B_{1} / B_{2} / \ldots / B_{k}$ written in standard form by

$$
\operatorname{los}(\pi)=\left|B_{2}\right|+2\left|B_{3}\right|+\cdots+(k-1)\left|B_{k}\right| .
$$

It is easy to see that the parameter los is a Z-statistic. Indeed, for a 2-partition $B_{1} / B_{2}$ written in standard form, we have $\operatorname{los}\left(B_{1} / B_{2}\right)=\left|B_{2}\right|$ whence for $\pi=B_{1} / B_{2} / \ldots / B_{k}$ we have

$$
\sum_{1 \leq i<j \leq k} \operatorname{STAT}\left(\operatorname{st}\left(B_{i} / B_{j}\right)\right)=\sum_{1 \leq i<j \leq k}\left|B_{j}\right|=\sum_{j=2}^{k}(j-1)\left|B_{j}\right|=\operatorname{los}(\pi)
$$

\subsubsection{Number of linear crossings}

One of the most widely occurring property of set partitions is the "noncrossing" property. This is essentially due to the important number of works on noncrossing partitions which appear in different fields of mathematics. The terminology originated probably from the graphical representation of set partitions (see e.g. [33]). The linear representation of a partition of a set $S \subseteq \mathbb{P}$ is obtained as follows: draw the elements of $S$ in increasing order on a line and join successive elements of each block by arcs drawn in the upper half-plane.

Alternatively, the elements may be represented on a circle and circularly successive elements of each block are joined by chords: this is the circular representation. For instance, the linear and circular representations of the partition $\pi=110 / 2379 / 4 / 5612 / 811$ are given in Figure 1. Linear and circular representations of set partitions suggest many statistics. The most studied is the number of crossings, but other statistics like nestings, alignments, $k$-distant crossings, $k$-crossings, $k$-nestings, etc. have also appeared in the literature (see e.g. $[4,6,22]$ ).

For a set partition $\pi \in \Pi$, we will denote by $\mathrm{cr}^{(\ell)}(\pi)$ the number of crossings in the linear representation of $\pi$. We will refer to such crossings as linear crossings, in order to distinguish these from the circular case. Formally, a linear crossing is a sequence 
$\left(i_{1}, j_{1}\right),\left(i_{2}, j_{2}\right)$ of edges of $\pi$, such that $i_{1}<i_{2}<j_{1}<j_{2}$. Here, an edge of $\pi$ is just a pair $(i, j)$ of consecutive elements $i<j$ in the same block $B$, where consecutive means that there is no element $p \in B$ such that $i<p<j$. For instance, if $\pi$ is the set partition represented in Figure 1, then $\mathrm{cr} r^{(\ell)}(\pi)=4$. In order to show that $\mathrm{cr} r^{(\ell)}$ is a Z-statistic, it suffices essentially to observe that a linear crossing is composed of two edges from distinct blocks (details are left to the reader).

Theorem 2.3. The average number $\mu_{n}$ of linear crossings in a random set partition of $[n]$ satisfies

$$
\begin{aligned}
\mu_{n} & =-\frac{5}{4} \frac{B_{n+2}}{B_{n}}+\left(\frac{n}{2}+\frac{9}{4}\right) \frac{B_{n+1}}{B_{n}}+\frac{n}{2}+\frac{1}{4} \\
& =\frac{n^{2}}{2 \log n}\left(1+\frac{\log \log n}{\log n}(1+o(1))\right) \quad \text { as } n \rightarrow \infty .
\end{aligned}
$$

The average number $\mu_{n, k}$ of linear crossings in a random set partition of $[n]$ into $k$ blocks satisfies

$$
\begin{aligned}
\mu_{n, k} & =\frac{1}{2} n(k-1)-\frac{5}{2}\left(\begin{array}{l}
k \\
2
\end{array}\right)+\frac{3}{2}(n+1-k) \frac{S_{n, k-1}}{S_{n, k}} \\
& =\frac{1}{2} n(k-1)-\frac{5}{2}\left(\begin{array}{l}
k \\
2
\end{array}\right)+o(1) \quad \text { as } n \rightarrow \infty .
\end{aligned}
$$

\subsubsection{Number of circular crossings}

For a set partition $\pi \in \Pi$, we will denote by $\mathrm{cr}^{(c)}(\pi)$ the number of crossings in the circular representation of $\pi$. We will refer to such crossings as circular crossings. Formally, a circular crossing is a sequence $\left(i_{1}, j_{1}\right),\left(i_{2}, j_{2}\right)$ of "circular edges" of $\pi$, such that $i_{1}<i_{2}<j_{1}<j_{2}$. Here, a circular edge of $\pi$ is a pair $(i, j)$ such that $i, j$ are consecutive elements in the same block of $\pi$ or $i=\min (B)$ and $j=\max (B)$ for some block $B \in \pi$.

For instance, if $\pi$ is the set partition represented in Figure 1, then $\mathrm{cr}(c)(\pi)=9$. We leave the verification that $\mathrm{cr}^{(c)}$ is a Z-statistic to the reader.

Theorem 2.4. The average number $\mu_{n}$ of circular crossings in a random set partition of $[n]$ satisfies

$$
\begin{aligned}
\mu_{n} & =\frac{n}{2} \frac{B_{n+1}}{B_{n}}+\frac{3 n}{2}-\frac{n(4 n+1)}{2} \frac{B_{n-1}}{B_{n}}-\left(\begin{array}{c}
n \\
2
\end{array}\right) \frac{B_{n-2}}{B_{n}}+\left(\begin{array}{l}
n \\
4
\end{array}\right) \frac{B_{n-4}}{B_{n}} \\
& =\frac{n^{2}}{2 \log n}\left(1+\frac{\log \log n}{\log n}(1+o(1))\right) \quad \text { as } n \rightarrow \infty .
\end{aligned}
$$

The average number $\mu_{n, k}$ of circular crossings in a random set partition of $[n]$ into $k$ blocks 
satisfies

$$
\begin{aligned}
\mu_{n, k} & =\frac{1}{2} n(k-1)-\frac{1}{2} n(4 n-5 k+1) \frac{S_{n-1, k-1}}{S_{n, k}}-10\left(\begin{array}{l}
n \\
2
\end{array}\right) \frac{S_{n-2, k-2}}{S_{n, k}} \\
& +\left(\begin{array}{c}
n \\
4
\end{array}\right) \frac{S_{n-4, k-2}}{S_{n, k}} \\
& =\frac{1}{2} n(k-1)+o(1) \quad \text { as } n \rightarrow \infty .
\end{aligned}
$$

\subsubsection{Number of overlappings}

Flajolet and Schott [12] considered a special class of set partitions, called non-overlapping partitions. With the implied order structure, two sets $B$ and $B^{\prime}$ are said to overlap if $\min (B)<\min \left(B^{\prime}\right)<\max (B)<\max \left(B^{\prime}\right)$. Then, a partition is said to be non-overlapping if any two blocks do not overlap. This property of non-overlapping could be seen graphically by considering the line diagram (see Figure 1) of a set partition. This suggests to consider statistics like the numbers of overlappings, embracings ("nestings of blocks"), etc.

The number of overlappings of a set partition $\pi$ will be denoted ovl $(\pi)$. For instance, if $\pi$ is the set partition represented in Figure 1, then $\operatorname{ovl}(\pi)=4$. We leave the verification that ovl is a Z-statistic to the reader.

Theorem 2.5. The average number $\mu_{n}$ of overlappings in a random set partition of $[n]$ satisfies

$$
\begin{aligned}
\mu_{n} & =\frac{1}{4} \frac{B_{n+2}}{B_{n}}+\frac{3}{4} \frac{B_{n+1}}{B_{n}}-\left(n+\frac{5}{4}\right)-\frac{n}{2} \frac{B_{n-1}}{B_{n}} \\
& =\frac{1}{4}\left(\frac{n}{\log n}\right)^{2}\left(1+2 \frac{\log \log n}{\log n}(1+o(1))\right) \text { as } n \rightarrow \infty .
\end{aligned}
$$

The average number $\mu_{n, k}$ of overlappings in a random set partition of $[n]$ into $k$ blocks satisfies

$$
\begin{aligned}
\mu_{n, k} & =\frac{1}{2}\left(\begin{array}{l}
k \\
2
\end{array}\right)+n(k-1) \frac{S_{n-1, k-1}}{S_{n, k}}-\frac{3}{2}(n+k-1) \frac{S_{n, k-1}}{S_{n, k}} \\
& =\frac{1}{2}\left(\begin{array}{l}
k \\
2
\end{array}\right)+o(1) \quad \text { as } n \rightarrow \infty
\end{aligned}
$$

\subsubsection{Number of embracings}

In this section, we consider a natural partner for overlappings. Say that two sets $B$ and $B^{\prime}$ embrace if, with the implied order structure, $\min (B)<\min \left(B^{\prime}\right) \leq \max \left(B^{\prime}\right)<$ $\max (B)$. The number of embracings of a set partition $\pi$ will be denoted $\operatorname{emb}(\pi)$ and can be easily computed via the line diagram representation. For instance, if $\pi$ is the set partition represented in Figure 1, then $\operatorname{e} m b(\pi)=4$. We leave the verification that emb is a Z-statistic to the reader. 
Theorem 2.6. The average number $\mu_{n}$ of embracings in a random set partition of $[n]$ satisfies

$$
\begin{aligned}
\mu_{n} & =\frac{1}{4} \frac{B_{n+2}}{B_{n}}-\frac{5}{4} \frac{B_{n+1}}{B_{n}}+\frac{3}{4}+\frac{n}{2} \frac{B_{n-1}}{B_{n}} \\
& =\frac{1}{4}\left(\frac{n}{\log n}\right)^{2}\left(1+2 \frac{\log \log n}{\log n}(1+o(1))\right) \quad \text { as } n \rightarrow \infty
\end{aligned}
$$

The average number $\mu_{n, k}$ of embracings in a random set partition of $[n]$ into $k$ blocks satisfies

$$
\begin{aligned}
\mu_{n, k} & =\frac{1}{2}\left(\begin{array}{l}
k \\
2
\end{array}\right)-\frac{1}{2}(k-1) \frac{S_{n, k-1}}{S_{n, k}}+\frac{n}{2} \frac{S_{n-1, k-2}}{S_{n, k}} \\
& =\frac{1}{2}\left(\begin{array}{l}
k \\
2
\end{array}\right)+o(1) \quad \text { as } n \rightarrow \infty .
\end{aligned}
$$

\subsubsection{Number of occurrences of a 2-pattern}

We say that a word $\sigma=\sigma_{1} \sigma_{2} \ldots \sigma_{r}$ in $\{1,2\}^{*}$ is a 2-pattern if it contains the two letters 1 and 2. An occurrence of the 2-pattern $\sigma$ in a word $w=w_{1} w_{2} \ldots w_{n} \in \mathbb{P}^{*}$ is an $r$-tuple $\left(i_{1}, i_{2}, \ldots, i_{r}\right)$ such that $s t\left(w_{i_{1}} w_{i_{2}} \ldots w_{i_{r}}\right)=\sigma$, where $s t$ is the standardization map (see Section 2.2). For instance, in the word $w=2431226737$, the 3-tuples $(1,3,9),(4,5,6)$ and $(7,8,10)$ are occurrences of the pattern 122 , while the 3 -tuples $(2,3,9)$ and $(3,5,6)$ are occurrences of the pattern 211 . The number of occurrences of a pattern $\sigma$ in a word $w$ will be denoted $\operatorname{occ}_{\sigma}(w)$. Since a set partition can be identified with its restricted growth function, we will set $\operatorname{occ}_{\sigma}(\pi)=\operatorname{occ}_{\sigma}(w(\pi))$, where $w(\pi)$ is, as usual, the RGF associated to $\pi$. We leave the verification that $\mathrm{occ}_{\sigma}$ is a Z-statistic for any 2-pattern $\sigma$ to the reader.

Theorem 2.7. Let $\sigma=\sigma_{1} \sigma_{2} \ldots \sigma_{r}$ be a 2-pattern of length $r$, and let $\mu_{n}^{(\sigma)}$ denote the average number of occurrences of the pattern $\sigma$ in a random set partition of $[n]$.

(i) For fixed $n$, the average value $\mu_{n}^{(\sigma)}$ depends only on the first letter of $\sigma$.

For $i \in\{1,2\}$, denote by $\mu_{n}^{\left(\sigma_{1}=i\right)}$ the common value of $\mu_{n}^{(\tau)}$ for patterns $\tau$ (of length $r$ ) satisfying $\tau_{1}=i$.

(ii) For any integer $n \geq 1$, we have

$$
\mu_{n}^{\left(\sigma_{1}=1\right)}+\mu_{n}^{\left(\sigma_{1}=2\right)}=\left(\begin{array}{c}
n \\
r
\end{array}\right) \frac{B_{n+2-r}-B_{n+1-r}}{B_{n}} .
$$

For $j=0, \ldots, r$, set

$$
p_{j}(n)=\frac{(-1)^{r-j}}{2^{r-j+1}}\left(\left(\begin{array}{l}
n \\
j
\end{array}\right)+\frac{1}{2}\left(\begin{array}{c}
n \\
j-1
\end{array}\right)\right) .
$$


(iii) For any integer $n \geq 0$, we have

$$
\begin{aligned}
\mu_{n}^{\left(\sigma_{1}=2\right)=} & \sum_{j=0}^{r} p_{j}(n) \frac{B_{n+2-j}}{B_{n}}-\frac{1}{2}\left(\begin{array}{c}
n \\
r
\end{array}\right) \frac{B_{n+1-r}}{B_{n}}+\frac{(-1)^{r+1}}{2^{r+1}} \\
\mu_{n}^{\left(\sigma_{1}=1\right)=} & -\sum_{j=0}^{r-1} p_{j}(n) \frac{B_{n+2-j}}{B_{n}}+\frac{1}{2}\left(\left(\begin{array}{l}
n \\
r
\end{array}\right)-\frac{1}{2}\left(\begin{array}{c}
n \\
r-1
\end{array}\right)\right) \frac{B_{n+2-r}}{B_{n}} \\
& -\frac{1}{2}\left(\begin{array}{l}
n \\
r
\end{array}\right) \frac{B_{n+1-r}}{B_{n}}+\frac{(-1)^{r}}{2^{r+1}} .
\end{aligned}
$$

(iv) For any 2-pattern $\sigma$ of length $r$, we have the asymptotic approximation

$$
\mu_{n}^{(\sigma)}=\frac{1}{2 r !} n^{2}(\log n)^{r-2}\left(1-(r-2) \frac{\log \log n}{\log n}+o\left(\frac{\log \log n}{\log n}\right)\right), \quad n \rightarrow \infty .
$$

(v) For the 2-patterns $\sigma$ of length 2, i.e., $\sigma \in\{12,21\}$, we have the more precise asymptotic approximation

$$
\mu_{n}^{(\sigma)}=\frac{n^{2}}{4}\left(1-\frac{1}{\log n}-\frac{\log \log n}{(\log n)^{2}}(1+o(1))\right), \quad n \rightarrow \infty .
$$

To illustrate our result, we give the values of $\mu_{n}^{(\sigma)}$ for 2-patterns $\sigma$ of length $r \leq 3$. For patterns of length $r=2$, we have

$$
\begin{aligned}
& \mu_{n}^{(21)}=\frac{1}{8} \frac{B_{n+2}}{B_{n}}-\left(\frac{n}{4}+\frac{1}{8}\right) \frac{B_{n+1}}{B_{n}}+\left(\frac{n^{2}}{4}-\frac{1}{8}\right)-\frac{1}{2}\left(\begin{array}{l}
n \\
2
\end{array}\right) \frac{B_{n-1}}{B_{n}} \\
& \mu_{n}^{(12)}=-\frac{1}{8} \frac{B_{n+2}}{B_{n}}+\left(\frac{n}{4}+\frac{1}{8}\right) \frac{B_{n+1}}{B_{n}}+\left(\frac{n^{2}}{4}-\frac{n}{2}+\frac{1}{8}\right)-\frac{1}{2}\left(\begin{array}{l}
n \\
2
\end{array}\right) \frac{B_{n-1}}{B_{n}} .
\end{aligned}
$$

For patterns of length $r=3$, we have

$$
\begin{aligned}
\mu_{n}^{(211)}=\mu_{n}^{(212)}=\mu_{n}^{(221)}=- & \frac{1}{16} \frac{B_{n+2}}{B_{n}}+\frac{1}{8}\left(n+\frac{1}{2}\right) \frac{B_{n+1}}{B_{n}}-\frac{1}{8}\left(n^{2}-\frac{1}{2}\right) \\
& +\frac{1}{12} n\left(n-\frac{1}{2}\right)(n-1) \frac{B_{n-1}}{B_{n}}-\frac{1}{2}\left(\begin{array}{c}
n \\
3
\end{array}\right) \frac{B_{n-2}}{B_{n}} \\
\mu_{n}^{(112)}=\mu_{n}^{(121)}=\mu_{n}^{(122)}=\frac{1}{16} & \frac{B_{n+2}}{B_{n}}-\frac{1}{8}\left(n+\frac{1}{2}\right) \frac{B_{n+1}}{B_{n}}+\frac{1}{8}\left(n^{2}-\frac{1}{2}\right) \\
& +\frac{1}{12} n(n-1)\left(n-\frac{7}{2}\right) \frac{B_{n-1}}{B_{n}}-\frac{1}{2}\left(\begin{array}{c}
n \\
3
\end{array}\right) \frac{B_{n-2}}{B_{n}} .
\end{aligned}
$$

Theorem 2.8. Let $\sigma=\sigma_{1} \sigma_{2} \ldots \sigma_{r}$ be a 2-pattern of length $r$, and let $\mu_{n, k}^{(\sigma)}$ denote the average number of occurrences of the pattern $\sigma$ in a random set partition of $[n]$ into $k$ blocks. 
(i) For fixed $n$ and $k$, the average value $\mu_{n, k}^{(\sigma)}$ depends only on the first letter of $\sigma$.

For $i \in\{1,2\}$, denote by $\mu_{n, k}^{\left(\sigma_{1}=i\right)}$ the common value of $\mu_{n, k}^{(\tau)}$ for patterns $\tau$ (of length $r$ ) satisfying $\tau_{1}=i$.

(ii) For any integer $n \geq k \geq 1$, we have

$$
\mu_{n, k}^{\left(\sigma_{1}=1\right)}+\mu_{n, k}^{\left(\sigma_{1}=2\right)}=\left(\begin{array}{c}
n \\
r
\end{array}\right) \frac{S_{n+2-r, k}-S_{n+1-r, k}}{S_{n, k}} .
$$

(iii) For any integer $n \geq k \geq 1$, we have

$$
\begin{aligned}
\mu_{n, k}^{\left(\sigma_{1}=2\right)}= & \sum_{j=0}^{r} p_{j}(n) \frac{S_{n+2-j, k}}{S_{n, k}}-\frac{1}{2}\left(\begin{array}{c}
n \\
r
\end{array}\right) \frac{S_{n+1-r, k}}{S_{n, k}}+\frac{(-1)^{r+1}}{2^{r+1}} \frac{S_{n, k-2}}{S_{n, k}} \\
\mu_{n, k}^{\left(\sigma_{1}=1\right)}= & -\sum_{j=0}^{r-1} p_{j}(n) \frac{S_{n+2-j, k}}{S_{n, k}}+\frac{1}{2}\left(\left(\begin{array}{c}
n \\
r
\end{array}\right)-\frac{1}{2}\left(\begin{array}{c}
n \\
r-1
\end{array}\right)\right) \frac{S_{n+2-r, k}}{S_{n, k}} \\
& -\frac{1}{2}\left(\begin{array}{c}
n \\
r
\end{array}\right) \frac{S_{n+1-r, k}}{S_{n, k}}+\frac{(-1)^{r}}{2^{r+1}} \frac{S_{n, k-2}}{S_{n, k}}
\end{aligned}
$$

where $p_{j}(n)$ is given by (2.24).

(iv) As $n \rightarrow \infty$, we have the following asymptotic approximations

$$
\begin{aligned}
& \mu_{n, k}^{\left(\sigma_{1}=2\right)}=\sum_{j=0}^{r} p_{j}(n) k^{2-j}-\frac{1}{2}\left(\begin{array}{l}
n \\
r
\end{array}\right) k^{1-r}+o(1) \\
& \mu_{n, k}^{\left(\sigma_{1}=1\right)}=-\sum_{j=0}^{r} p_{j}(n) k^{2-j}+\left(\begin{array}{l}
n \\
r
\end{array}\right) k^{2-r}-\frac{1}{2}\left(\begin{array}{l}
n \\
r
\end{array}\right) k^{1-r}+o(1) .
\end{aligned}
$$

In particular, for any 2-pattern $\sigma$ of length $r$, we have

$$
\mu_{n, k}^{(\sigma)} \sim \frac{n^{r}}{2 k^{r-1} r !}(k-1) \quad \text { as } n \rightarrow \infty .
$$

\subsection{Plan of the paper}

In the next section, we will prove the key result of the paper (Theorem 3.1), which expresses average values of Z-statistics as coefficients in certain generating functions. Moreover, there we introduce the tools which are necessary to use our key result. The purpose of the other sections (except the last) is to prove the results presented in Section 2. Finally, we will conclude the paper with some remarks, notably that the methodology could be adapted to other exponential families and to more general statistics. 


\section{Average value of a $\mathrm{Z}$-statistic in a random set par- tition}

We said in the previous section that the computation of the average value of a Z-statistic in a random set partition is essentially equivalent to the computation of its average value in a random 2-partition. Let us make this more precise. Given a Z-statistic stat, let $v_{n, 2}^{\text {stat }}:=\sum_{\pi \in \Pi_{n}^{2}} \operatorname{stat}(\pi)$, and let $V_{2}^{\text {stat }}(x)$ be the exponential generating function of the sequence $\left(v_{n, 2}^{\text {stat }}\right)_{n \geq 0}$, i.e.,

$$
V_{2}^{\text {stat }}(x)=\sum_{n \geq 0} v_{n, 2}^{\text {stat }} \frac{x^{n}}{n !}=\sum_{n \geq 0} \sum_{\pi \in \Pi_{n}^{2}} \operatorname{stat}(\pi) \frac{x^{n}}{n !} .
$$

The following theorem is the key result of the paper.

Theorem 3.1. Let stat be a Z-statistic. Denote by $\mu_{n}\left(\mu_{n, k}\right.$, respectively) the average value of the statistic stat in a random set partition of $[n]$ (respectively of $[n]$ into $k$ blocks). Then we have

$$
\begin{aligned}
\mu_{n} & =\frac{1}{B_{n}}\left[\frac{x^{n}}{n !}\right] V_{2}^{\text {stat }}(x) B(x) \\
\mu_{n, k} & =\frac{1}{S_{n, k}}\left[\frac{x^{n}}{n !}\right] V_{2}^{\text {stat }}(x) S_{k-2}(x),
\end{aligned}
$$

where $B_{n}$ and $S_{n, k}$ are Bell and Stirling numbers, and $B(x)$ and the $S_{j}(x)$ 's are exponential generating functions of Bell and Stirling numbers defined in (3.6) and (3.8).

After coefficient extraction, Theorem 3.1 can lead to exact and asymptotic formulas for average values of Z-statistics. For this purpose, we need some tools that we develop in the next subsection. It is worth noting that, here, by an exact formula, we mean a finite expression which involves elementary functions, and Bell and Stirling numbers. We then present a process to get the average and asymptotic value of Z-statistics. In a particular case (which includes all the statistics we consider in this paper), the algorithm is deterministic. We will illustrate the process in the third subsection. Finally, we will prove our key result, Theorem 3.1, in the last subsection.

\subsection{Basic tools}

\subsubsection{Basic properties of Stirling and Bell numbers}

Recall that the number of $k$-partitions of an $n$-set is the Stirling number of the second kind $S_{n, k}$, and the number of all partitions of an $n$-set is the Bell number $B_{n}$, so that we have $B_{n}=\sum_{k=1}^{n} S_{n, k}$. Classifying the $k$-partitions of an $n$-set as to whether they do or do not contain a given element yields the recurrence:

$$
S_{n, k}=S_{n-1, k-1}+k S_{n-1, k} \quad(n \geq k \geq 1),
$$


with $S_{j, 0}=S_{0, j}=\delta_{j, 0}$.

As noted previously, the number of surjections from an $n$-set $N$ to a $k$-set $K$ is equal to $k ! S_{n, k}$. Then, given a set $X,|X|=x$, classifying all the maps $f: N \rightarrow X$ according to the cardinality of their image leads to the fundamental identity

$$
x^{n}=\sum_{k=0}^{n}\left(\begin{array}{l}
x \\
k
\end{array}\right) k ! S_{n, k}=\sum_{k=0}^{n} S_{n, k}(x)_{k},
$$

from which, by binomial inversion, we deduce the summation formula

$$
S_{n, k}=\frac{1}{k !} \sum_{j=0}^{k}(-1)^{j}\left(\begin{array}{l}
k \\
j
\end{array}\right)(k-j)^{n} .
$$

Here and in the rest of the paper, $(x)_{k}$ stands for the $k$-th lower factorial $x(x-1) \cdots(x-$ $k+1$ ). Equation (3.5) leads to the generating function identity

$$
S_{k}(t):=\sum_{n \geq 0} S_{n, k} \frac{t^{n}}{n !}=\frac{1}{k !}\left(e^{t}-1\right)^{k},
$$

from which we deduce the double generating function

$$
F(t, u):=\sum_{n \geq k \geq 0} S_{n, k} u^{k} \frac{t^{n}}{n !}=\sum_{k \geq 0} S_{k}(t) u^{k}=e^{u\left(e^{t}-1\right)} .
$$

Setting $u=1$, we recover the exponential generating function of Bell numbers

$$
B(t):=\sum_{n \geq 0} B_{n} \frac{t^{n}}{n !}=e^{e^{t}-1}
$$

There are of course many ways to recover all the previous identities. For instance, one could first obtain the double generating function (3.7) immediately from the exponential formula (a set partition is a "set of nonempty sets" in the language of species), and then by specializations, coefficient extractions, etc. recover all the previous results and even much more.

\subsubsection{Coefficient extraction}

Given a Z-statistic stat, the first step we have to perform in order to be able to apply Theorem 3.1 is to try to find a convenient expression for the power series $V_{2}^{\text {stat }}(x)$. It turns out that for all Z-statistics which the author was able to locate in the literature, the formal power series $V_{2}^{\text {stat }}(x)$ is given by

$$
V_{2}^{\text {stat }}(x)=P_{0}(x)+P_{1}(x) e^{x}+P_{2}(x) e^{2 x},
$$


where the $P_{i}$ are polynomials. Therefore, we need to develop some tools to cope with this situation. Elementary computations based on the formulas (3.8) and (3.6) show that we have

$$
\begin{aligned}
& e^{x} B(x)=B^{\prime}(x), \quad e^{2 x} B(x)=B^{\prime \prime}(x)-B^{\prime}(x) \\
& e^{x} S_{k-2}(x)=S_{k-2}(x)+(k-1) S_{k-1}(x) \\
& e^{2 x} S_{k-2}(x)=S_{k-2}(x)+2(k-1) S_{k-1}(x)+k(k-1) S_{k}(x) .
\end{aligned}
$$

This implies that, for $V_{2}^{\text {stat }}(x)$ satisfying (3.9), there exist polynomials $Q_{i}$ and $R_{i}$, $0 \leq i \leq 2$, such that

$$
\begin{aligned}
V_{2}^{\text {stat }}(x) B(x) & =Q_{0}(x) B(x)+Q_{1}(x) B^{\prime}(x)+Q_{2}(x) B^{\prime \prime}(x) \\
V_{2}^{\text {stat }}(x) S_{k-2}(x) & =R_{0}(x) S_{k-2}(x)+R_{1}(x) S_{k-1}(x)+R_{2}(x) S_{k}(x) .
\end{aligned}
$$

Suppose we are given a formal power series $A(x)=\sum_{n \geq 0} a_{n} \frac{x^{n}}{n !}$ and let $A^{(h)}(x)$ be the $h$-th formal derivative $A^{(h)}(x)$ of $A(x)$. Using the basic facts

$$
\left[\frac{x^{n}}{n !}\right] A^{(h)}(x)=a_{n+h} \quad\left[\frac{x^{n}}{n !}\right] x^{i} A(x)=(n)_{i} a_{n-i},
$$

coefficient extraction from power series of the form (3.13) or (3.14) becomes a routine process. In particular, if $V_{2}^{\text {stat }}(x)$ satisfies (3.9), it follows by Theorem 3.1 and (3.13)(3.15) that $\mu_{n}$ and $\mu_{n, k}$ are given by

$$
\mu_{n}=\sum_{i \in \mathbb{Z}} p_{i}(n) \frac{B_{n+i}}{B_{n}} \quad \text { and } \quad \mu_{n, k}=\sum_{i, j \geq 0} q_{i, j}(n) \frac{S_{n-i, k-j}}{S_{n, k}}
$$

where the $p_{i}(n)$ and $q_{i, j}(n)$ are polynomials in $n$ which are all zero except for a finite number of them.

\subsubsection{Asymptotics}

In order to obtain asymptotic approximations of the average values $\mu_{n}$ and $\mu_{n, k}$, in view of (3.16), it is important to consider quotients of Bell numbers and Stirling numbers. We begin with Stirling numbers.

It follows immediately from (3.5) that, as $n \rightarrow \infty$, we have

$$
S_{n, k}=\frac{k^{n}}{k !}+O\left((k-1)^{n}\right)=\frac{k^{n}}{k !}\left(1+O\left(\left(1-\frac{1}{k}\right)^{n}\right)\right),
$$

from which we deduce that, for $i, j \geq 0$, we have

$$
\begin{aligned}
\frac{S_{n-i, k-j}}{S_{n, k}} & =\frac{(k-j)^{n-i}}{(k-j) !}\left(1+O\left(\left(1-\frac{1}{k-j}\right)^{n-i}\right)\right) \frac{k !}{k^{n}}\left(1+O\left(\left(1-\frac{1}{k}\right)^{n}\right)\right)^{-1} \\
& =\frac{k !}{(k-j) !(k-j)^{i}}\left(1-\frac{j}{k}\right)^{n}\left(1+O\left(\left(1-\frac{1}{k}\right)^{n}\right)\right) .
\end{aligned}
$$

Distinct the cases $j=0$ and $j \geq 1$ leads to the following result. 
Lemma 3.2. For $i \geq 0$ and $j \geq 1$, we have, as $n \rightarrow \infty$,

$$
\begin{aligned}
\frac{S_{n-i, k}}{S_{n, k}} & =\frac{1}{k^{i}}+O\left(\left(1-\frac{1}{k}\right)^{n}\right) \\
\frac{S_{n-i, k-j}}{S_{n, k}} & =O\left(\left(1-\frac{j}{k}\right)^{n}\right) .
\end{aligned}
$$

The asymptotics of Bell numbers is more delicate and is best performed by a saddlepoint method. It is probably the most famous application of saddle-point techniques to combinatorial enumeration. The Bell number $B_{n}$ satisfies (see [13, Prop. VIII.3])

$$
B_{n}=n ! \frac{e^{e^{r}-1}}{r^{n} \sqrt{2 \pi r(r+1) e^{r}}}\left(1+O\left(e^{-r / 5}\right)\right),
$$

where $r$ is defined implicitly by $r e^{r}=n+1$, so that $r=(\log n) \cdot(\log \log n)+o(1)$. Instead of the latter formula, we will use the following corollary given by Salvy and Shackell [32]:

$$
\frac{B_{n+1}}{B_{n}}=\frac{n}{\log n}\left(1+\frac{\log \log n}{\log n}(1+o(1))\right) .
$$

Note that the latter identity was established because it gives an asymptotic approximation of the average number of blocks in a random set partition of $[n]$. It is also worth noting that there exists a more precise asymptotic approximation (see [32]) which the reader can use to get more precise asymptotic approximations for the average values computed in this paper.

Lemma 3.3. For any integer $r \in \mathbb{Z}$, we have, as $n \rightarrow \infty$,

$$
\frac{B_{n+r}}{B_{n}}=\left(\frac{n}{\log n}\right)^{r}\left(1+r \frac{\log \log n}{\log n}(1+o(1))\right) .
$$

Proof. Suppose we are given an integer $j$. It follows from (3.18) that

$$
\frac{B_{n+j+1}}{B_{n+j}}=\frac{n+j}{\log (n+j)}\left(1+\frac{\log \log (n+j)}{\log (n+j)}(1+o(1))\right) .
$$

By elementary calculus and the well-known formula $\log (1+x)=x(1+o(1))$ as $x \rightarrow 0$, it is easy to establish that

$$
\log (n+j)=\log n\left(1+O\left(\frac{1}{n \log n}\right)\right) \text { and } \frac{\log \log (n+j)}{\log (n+j)}=\frac{\log \log n}{\log n}(1+o(1)),
$$

and thus

$$
\frac{n+j}{\log (n+j)}=\frac{n\left(1+\frac{j}{n}\right)}{\log n}\left(1+O\left(\frac{1}{n \log n}\right)\right)^{-1}=\frac{n}{\log n}\left(1+O\left(\frac{1}{n}\right)\right) .
$$


Combining (3.20), (3.21), and (3.22), we obtain immediately

$$
\frac{B_{n+j+1}}{B_{n+j}}=\frac{n}{\log n}\left(1+\frac{\log \log n}{\log n}(1+o(1))\right),
$$

from which we deduce that, for any integer $r$, we have

$$
\begin{aligned}
\frac{B_{n+r}}{B_{n}} & =\prod_{j=0}^{r-1} \frac{B_{n+j+1}}{B_{n+j}}=\left(\left(\frac{n}{\log n}\right)\left(1+\frac{\log \log n}{\log n}(1+o(1))\right)\right)^{r} \\
& =\left(\frac{n}{\log n}\right)^{r}\left(1+r \frac{\log \log n}{\log n}(1+o(1))\right)
\end{aligned}
$$

since $(1+x)^{r}=1+r x+o(x)$ as $x \rightarrow 0$.

\section{$3.2 \quad$ A process}

By Theorem 3.1, in order to get the average values $\mu_{n}$ and $\mu_{n, k}$ of a Z-statistic stat, we can proceed essentially as follows:

(i) Compute $V_{2}^{\text {stat }}(x)$.

(ii) Extract coefficients from the power series $V_{2}^{\text {stat }}(x) B(x)$ and $V_{2}^{\text {stat }}(x) S_{k-2}(x)$.

Actually, the above process seems to be nondeterministic, since it is a priori not clear whether $V_{2}^{\text {stat }}(x)$ admits a simple expression (a finite expression involving elementary functions). However, as we mentioned previously, for all Z-statistics on set partitions which the author was able to locate in the literature, the formal power series $V_{2}^{\text {stat }}(x)$ admits the simple form (3.9). For such statistics, one can design a deterministic algorithm.

Input: a Z-statistic stat such that $V_{2}^{\text {stat }}(x)=P_{0}(x)+P_{1}(x) e^{x}+P_{2}(x) e^{2 x}$, where the $P_{i}$ are polynomials.

Output: (a) the exact average values $\mu_{n}$ and $\mu_{n, k}$, written as finite expressions which involve elementary functions, Bell and Stirling numbers; (b) asymptotic approximations of $\mu_{n}$ and $\mu_{n, k}$.

Step 1. Compute explicitly $V_{2}^{\text {stat }}(x)$ (i.e., determine the polynomials $P_{0}, P_{1}$ and $P_{2}$ ).

Step 2. Using (3.10)-(3.12), write $V_{2}^{\text {stat }}(x) B(x)$ and $V_{2}^{\text {stat }}(x) S_{k-2}(x)$ as

$$
\begin{aligned}
V_{2}^{\text {stat }}(x) B(x) & =Q_{0}(x) B(x)+Q_{1}(x) B^{\prime}(x)+Q_{2}(x) B^{\prime \prime}(x) \\
V_{2}^{\text {stat }}(x) S_{k-2}(x) & =R_{0}(x) S_{k-2}(x)+R_{1}(x) S_{k-1}(x)+R_{2}(x) S_{k}(x),
\end{aligned}
$$

where the $Q_{i}$ and $R_{i}$ are polynomials.

Step 3. Using (3.15), write the coefficients of $V_{2}^{\text {stat }}(x) B(x)$ and $V_{2}^{\text {stat }}(x) S_{k-2}(x)$ as

$$
\left[\frac{x^{n}}{n !}\right] V_{2}^{\text {stat }}(x) B(x)=\sum_{i \in \mathbb{Z}} p_{i}(n) B_{n+i} \text { and }\left[\frac{x^{n}}{n !}\right] V_{2}^{\text {stat }}(x) S_{k-2}=\sum_{i, j \geq 0} q_{i, j}(n, k) S_{n-i, k-j} \text {, }
$$


where the $p_{i}(n)$ and $q_{i, j}(n, k)$ are polynomials in $n$ and $k$ which are all zero except for a finite number of them.

Step 4. Divide the expressions obtained in the previous step by $B_{n}$ and $S_{n, k}$ in order to get the average values $\mu_{n}$ and $\mu_{n, k}$, which can be written in the form

$$
\left[\frac{x^{n}}{n !}\right] V_{2}^{\text {stat }}(x) B(x)=\sum_{i \in \mathbb{Z}} p_{i}(n) \frac{B_{n+i}}{B_{n}} \text { and }\left[\frac{x^{n}}{n !}\right] V_{2}^{\text {stat }}(x) S_{k-2}=\sum_{i, j \geq 0} q_{i, j}(n, k) \frac{S_{n-i, k-j}}{S_{n, k}} .
$$

Step 5. Use Lemmas 3.2 and 3.3 in the expressions obtained in the previous step to get asymptotic approximations of $\mu_{n}$ and $\mu_{n, k}$.

A proof that the above process is deterministic can be easily extracted from the previous subsection (details are left to the reader). To make things more concrete, we give an example in the next subsection.

\subsection{Example: an inversion statistic on set partitions}

In his study of some $q$-Stirling numbers which arose from a $q$-exponential formula, Johnson [17] (see also [8]) introduced an inversion statistic on set partitions, denoted here by inv, which can be defined as follows. For $\pi \in \Pi_{n}$ with standard form $\pi=B_{1} / B_{2} / \cdots B_{k}$, inv $(\pi)$ is the number of pairs $(i, j), 1 \leq i<j \leq n$, such that $i$ belongs to a block to the right of the block containing $j$. Equivalently, inv $(\pi)$ is the number of inversions in the RGF $w(\pi)$ of $\pi$. Recall that an inversion in a word $w_{1} \ldots w_{n}$ is a pair $(i, j), 1 \leq i<j \leq n$, such that $w_{i}>w_{j}$. For instance, there are exactly 3 inversions in $\pi=14 / 25 / 3 \equiv 12312=w(\pi)$ : the pairs $(2,4),(3,4)$ and $(3,5)$; hence $\mathrm{inv}(\pi)=3$. Note that inv $=\mathrm{occ}_{21}$ (where occ ${ }_{21}$ is defined in Section 3.3).

We propose to determine exact and asymptotic average numbers of inversions in a random set partition. Since inv is a Z-statistic, we must first "compute" $V_{2}^{\text {inv }}(x)$.

By definition, $V_{2}^{\text {inv }}(x)$ is the exponential generating function of the sequence $\left(v_{n}\right)_{n \geq 0}$ with $v_{n}:=v_{n, 2}^{\mathrm{i} n v}=\sum_{\pi \in \Pi_{n}^{2}} \mathrm{i} n v(\pi)$. For a combinatorialist, it is usual to interpret $v_{n}$ as

the number of pairs $(\pi, a)$, where $\pi$ is a 2-partition of $[n]$ and $a=(i, j)$ is an inversion of $\pi$. Such a pair will be called an underlined 2-partition. An underlined partition $(\pi, a)$ with $a=(i, j)$ can be identified with the restricted growth function $w(\pi)$ in which the letters $w_{i}, w_{j}$ are colored. For instance, there are 3 inversions in $\pi=14 / 25 / 3: a_{1}=(2,4)$, $a_{2}=(3,4)$ and $a_{3}=(3,5)$. Then we have $\left(\pi, a_{1}\right) \equiv 12312,\left(\pi, a_{2}\right) \equiv 12312$ and $\left(\pi, a_{3}\right) \equiv 12312$.

Then, using this correspondence, it is not hard to see that an underlined 2-partition $(\pi, a)$ can be uniquely written as

$$
(\pi, a) \equiv 1 u_{1} 2 u_{2} \mathbf{1} u_{3}
$$

with $u_{1}, u_{2}, u_{3} \in\{1,2\}^{*}$. Here and in the rest of the paper, we write $X^{*}$ for the free monoid generated by an alphabet $X$, which is the set of words whose letters are in $X$. The empty word will be denoted $\epsilon$. 
Elementary counting (there are $\left(\begin{array}{c}n-1 \\ 2\end{array}\right)$ choices for the positions of the underlined elements and $2^{n-3}$ choices for the word $u_{1} u_{2} u_{3}$ ) leads to

$$
v_{n}= \begin{cases}\left(\begin{array}{c}
n-1 \\
2
\end{array}\right) 2^{n-3}, & \text { if } n \geq 2 \\
0, & \text { if } n \leq 1\end{cases}
$$

A straightforward computation, the details of which are left to the reader, then yields

$$
V_{2}^{\mathrm{inv}}(x)=\sum_{n \geq 0} v_{n} \frac{x^{n}}{n !}=\left(\frac{x^{2}}{4}-\frac{x}{4}+\frac{1}{8}\right) e^{2 x}-\frac{1}{8} .
$$

Since $V_{2}^{\text {inv }}(x)$ can be written in the form (3.9), we can continue the process described in the previous subsection.

Step 2. Using (3.10) and (3.12), it is straightforward to obtain

$$
\begin{gathered}
V_{2}^{\mathrm{i} n v}(x) B(x)=-\frac{1}{8} B(x)+\frac{1}{8}\left(-1+2 x-2 x^{2}\right) B^{\prime}(x)+\frac{1}{8}\left(1-2 x+2 x^{2}\right) B^{\prime \prime}(x) \\
V_{2}^{\mathrm{i} n v}(x) S_{k-2}(x)=\frac{1}{4}\left(-x+x^{2}\right) S_{k-2}(x)+\frac{1}{4}(k-1)\left(1-2 x+2 x^{2}\right) S_{k-1}(x) \\
+\frac{1}{8} k(k-1)\left(1-2 x+2 x^{2}\right) S_{k}(x) .
\end{gathered}
$$

Step 3. After routine coefficient extraction based on (3.15), we obtain

$$
\begin{aligned}
{\left[\frac{x^{n}}{n !}\right] V_{2}^{\mathrm{inv}}(x) B(x)=} & \frac{1}{8} B_{n+2}-\left(\frac{n}{4}+\frac{1}{8}\right) B_{n+1}+\left(\frac{n^{2}}{4}-\frac{1}{8}\right) B_{n}-\frac{(n)_{2}}{4} B_{n-1} \\
{\left[\frac{x^{n}}{n !}\right] V_{2}^{\mathrm{i} n v}(x) S_{k-2}(x)=\frac{1}{4} } & \left(-n S_{n-1, k-2}+(n)_{2} S_{n-2, k-2}\right) \\
+ & \frac{1}{4}(k-1)\left(S_{n, k-1}-2 n S_{n-1, k-1}+2(n)_{2} S_{n-2, k-1}\right) \\
+ & \frac{1}{8} k(k-1)\left(S_{n, k}-2 n S_{n-1, k}+2(n)_{2} S_{n-2, k}\right) .
\end{aligned}
$$

It is possible to simplify the expression obtained for $\left[\frac{x^{n}}{n !}\right] V_{2}^{\text {inv }}(x) S_{k-2}(x)$. First, replace each occurrence of the left hand sides of the three identities

$$
\begin{aligned}
S_{n-2, k-2} & =S_{n-1, k-1}-(k-1) S_{n-2, k-1} \\
S_{n-1, k-2} & =S_{n, k-1}-(k-1) S_{n-1, k-1} \\
S_{n-2, k} & =\frac{1}{k}\left(S_{n-1, k}-S_{n-2, k-1}\right)
\end{aligned}
$$

by the corresponding right hand sides. Then, in the identity obtained from the previous manipulation, replace each occurrence of the left hand side of the identity

$$
S_{n-1, k}=\frac{1}{k}\left(S_{n, k}-S_{n-1, k-1}\right)
$$


by its right hand side. This gives

$$
\begin{aligned}
{\left[\frac{x^{n}}{n !}\right] V_{2}^{\mathrm{inv}}(x) S_{k-2}(x)=} & \left(\frac{1}{4} n(n-k-1)\left(1-\frac{1}{k}\right)+\frac{1}{8} k(k-1)\right) S_{n, k} \\
& -\frac{1}{4}(n+1-k) S_{n, k-1}+\frac{n(n-1)}{4 k} S_{n-1, k-1} .
\end{aligned}
$$

Note that identities (3.25)-(3.28) could be obtained in an elementary way from (3.4).

Steps 4 and 5. Divide the expression (3.24) by $B_{n}$ to obtain that the average number of inversions $\mu_{n}$ in a random set partition of $[n]$ is given by

$$
\mu_{n}=\frac{1}{8} \frac{B_{n+2}}{B_{n}}-\left(\frac{n}{4}+\frac{1}{8}\right) \frac{B_{n+1}}{B_{n}}+\left(\frac{n^{2}}{4}-\frac{1}{8}\right)-\frac{(n)_{2}}{4} \frac{B_{n-1}}{B_{n}} .
$$

Using Lemma 3.3, it is straightforward to arrive at the asymptotic approximation

$$
\mu_{n}=\frac{n^{2}}{4}\left(1-\frac{1}{\log n}-\frac{\log \log n}{(\log n)^{2}}(1+o(1))\right), \quad n \rightarrow \infty .
$$

Similarly, divide expression (3.29) by $S_{n, k}$ to obtain that the average number of inversions $\mu_{n, k}$ in a random set partition of $[n]$ into $k$ blocks is given by

$$
\begin{aligned}
\mu_{n, k}=\frac{1}{4} & n(n-k-1)\left(1-\frac{1}{k}\right)+\frac{1}{8} k(k-1)-\frac{1}{4}(n+1-k) \frac{S_{n, k-1}}{S_{n, k}} \\
+ & \frac{n(n-1)}{4 k} \frac{S_{n-1, k-1}}{S_{n, k}} .
\end{aligned}
$$

Use of Lemma 3.2 then produces the asymptotic approximation

$$
\begin{aligned}
\mu_{n, k} & =\frac{1}{4} n(n-k-1)\left(1-\frac{1}{k}\right)+\frac{1}{8} k(k-1)+O\left(n\left(1-\frac{1}{k}\right)^{n}\right) \\
& =\frac{1}{4} n(n-k-1)\left(1-\frac{1}{k}\right)+\frac{1}{8} k(k-1)+o(1), \quad \text { as } n \rightarrow \infty .
\end{aligned}
$$

\subsection{Proof of Theorem 3.1}

Let stat be a Z-statistic defined on set partitions. It is easy to see that (3.2) is a corollary of (3.3). Indeed, by definition of the average value, we have

$$
\mu_{n}=\frac{\sum_{\pi \in \Pi_{n}} \operatorname{stat}(\pi)}{B_{n}}=\frac{\sum_{k \geq 1} \sum_{\pi \in \Pi_{n}^{k}} \operatorname{stat}(\pi)}{B_{n}}=\frac{1}{B_{n}} \sum_{k \geq 1} S_{n, k} \mu_{n, k} .
$$


Suppose that (3.3) is true. Then we have

$$
\begin{aligned}
\mu_{n} & =\frac{1}{B_{n}} \sum_{k \geq 1} S_{n, k} \mu_{n, k}=\frac{1}{B_{n}} \sum_{k \geq 1}\left[\frac{x^{n}}{n !}\right] V_{2}^{\text {stat }}(x) S_{k-2}(x) \\
& =\frac{1}{B_{n}}\left[\frac{x^{n}}{n !}\right] V_{2}^{\text {stat }}(x)\left(\sum_{k \geq 1} S_{k-2}(x)\right)=\frac{1}{B_{n}}\left[\frac{x^{n}}{n !}\right] V_{2}^{\text {stat }}(x) B(x) .
\end{aligned}
$$

Therefore, in order to prove Theorem 3.1, we just have to prove (3.3). Set $v_{m}:=v_{m, 2}^{\text {stat }}$. By the definitions of average value and product of two formal power series, Equation (3.3) is equivalent to

$$
\sum_{\pi \in \Pi_{n}^{k}} \operatorname{stat}(\pi)=\sum_{m=0}^{n}\left(\begin{array}{c}
n \\
m
\end{array}\right) S_{n-m, k-2} v_{m}
$$

We will prove the latter identity. Let $D I S J$ be the set of pairs of nonempty and disjoints subsets of $\mathbb{P}$, i.e.,

$$
D I S J=\{\{A, B\} / A, B \subseteq \mathbb{P}, A, B \neq \emptyset, A \cap B=\emptyset\} .
$$

Then, by definition of a Z-statistic, we have

$$
\begin{aligned}
\sum_{\pi \in \Pi_{n}^{k}} \operatorname{stat}(\pi) & =\sum_{\pi \in \Pi_{n}^{k}} \sum_{A, B \in \pi} \operatorname{stat}(\operatorname{st}(\{A, B\}))=\sum_{\substack { A, B \subseteq[n] \\
\begin{subarray}{c}{\pi \in \Pi_{n}^{k} \\
A, B \in \pi{ A , B \subseteq [ n ] \\
\begin{subarray} { c } { \pi \in \Pi _ { n } ^ { k } \\
A , B \in \pi } }\end{subarray}} \operatorname{stat}(\operatorname{st}(\{A, B\})) \\
& =\sum_{\substack{A, B \subseteq[n] \\
\{A, B\} \in D I S J}} \operatorname{stat}(\operatorname{st}(\{A, B\})) S_{n-|A \cup B|, k-2,}
\end{aligned}
$$

where the last equality follows from the fact that, for $A, B \subseteq[n]$, the number $p_{n, k}(A, B)$ of partitions $\pi$ in $\Pi_{n}^{k}$ such that $A, B \in \pi$ is given by

$$
p_{n, k}(A, B)= \begin{cases}S_{n-|A \cup B|, k-2}, & \text { if }\{A, B\} \in D I S J \\ 0, & \text { otherwise. }\end{cases}
$$

Now, suppose we are given a set $C \subseteq \mathbb{P}$ with $|C|=m$. We have

$$
\sum_{\substack{\{A, B\} \in D I S J \\ A \cup B=C}} \operatorname{stat}(\mathrm{st}(\{A, B\}))=\sum_{\tau \in \Pi^{2}(C)} \operatorname{stat}\left(\mathrm{st} t_{C}(\tau)\right)=\sum_{\pi \in \Pi_{m}^{2}} \operatorname{stat}(\pi)=v_{m}
$$

where the first equality follows from the definition of $D I S J$ and the second is a consequence of the fact that $s t_{C}$ send $\Pi^{2}(C)$ bijectively onto $\Pi_{m}^{2}$. 
Combining (3.32) and (3.33), we obtain

$$
\begin{aligned}
\sum_{\pi \in \Pi_{n}^{k}} \operatorname{stat}(\pi) & =\sum_{\substack{A, B \subseteq[n] \\
\{A, B\} \in D I S J}} \operatorname{stat}(\operatorname{st}(\{A, B\})) S_{n-|A \cup B|, k-2} \\
& =\sum_{C \subseteq[n]} \sum_{\substack{\{A, B\} \in D I S J \\
A \cup B=C}} \operatorname{stat}(\operatorname{st}(\{A, B\})) S_{n-|A \cup B|, k-2} \\
& =\sum_{C \subseteq[n]} v_{|C|} S_{n-|C|, k-2},
\end{aligned}
$$

which is obviously equivalent to (3.31). This concludes the proof.

Remark 3.1. Using the language of what is called the theory of species one can give a quick proof of Theorem 3.1. We have preferred to avoid this terminology in order to keep the paper self-contained.

\section{Carlitz's $q$-Stirling distributions}

\subsection{Proof of Theorem 2.2}

We will work here with the Z-statistic los, which has the $q$-Stirling distribution $S(q)$ (see $[27,34])$. Recall that los can be defined for a set partition $\pi=B_{1} / B_{2} / \ldots / B_{k}$ in standard form by

$$
\operatorname{los}(\pi)=\left|B_{2}\right|+2\left|B_{3}\right|+\cdots+(k-1)\left|B_{k}\right| .
$$

We have to start by "computing" $V_{2}^{\text {los }}(x)$.

Computation of $V_{2}^{\text {los }}(x)$. By definition, $V_{2}^{\text {los }}(x)$ is the exponential generating function of the sequence $\left(v_{n}\right)_{n \geq 0}$ with $v_{n}:=v_{n, 2}^{\text {los }}=\sum_{\pi \in \Pi_{n}^{2}} \operatorname{los}(\pi)$.

For a 2-partition $\pi=B_{1} / B_{2}$ written in standard form, we have $\operatorname{los}(\pi)=\left|B_{2}\right|$. Thus, we can see $v_{n}$ as the number of pairs $(\pi, i)$, where $\pi=B_{1} / B_{2}$ is a 2-partition of $[n]$ (written in standard form) and $i \in B_{2}$. The number of such pairs is easily shown to be $(n-1) 2^{n-2}$. Indeed, we have $n-1$ choices for the element $i$ (any of the elements of $\{2,3, \ldots, n\}$ ) and then $2^{n-2}$ choices for the set $B_{2} \backslash\{i\}$ (any of the subsets of $\{2,3, \ldots, n\} \backslash\{i\}$ ). Therefore, we have

$$
v_{n}=\sum_{\pi \in \Pi_{n}^{2}} \operatorname{los}(\pi)= \begin{cases}0, & \text { if } n \leq 1 \\ (n-1) 2^{n-2}, & \text { if } n \geq 2\end{cases}
$$

A straightforward computation leads to

$$
V_{2}^{\text {los }}(x)=\sum_{n \geq 0} v_{n} \frac{x^{n}}{n !}=\sum_{n \geq 2}(n-1) 2^{n-2} \frac{x^{n}}{n !}=\frac{1}{4}+\left(-\frac{1}{4}+\frac{x}{2}\right) e^{2 x} .
$$


Since $V_{2}^{\text {los }}(x)$ can be written in the form (3.9), we can continue the process described in Section 3.2.

Step 2. Using (3.10) and (3.12), we can write

$$
\begin{aligned}
V_{2}^{\text {los }}(x) B(x) & =\frac{1}{4} B(x)+\left(-\frac{x}{2}+\frac{1}{4}\right) B^{\prime}(x)+\left(\frac{x}{2}-\frac{1}{4}\right) B^{\prime \prime}(x) \\
V_{2}^{\text {los }}(x) S_{k-2}(x) & =\frac{x}{2} S_{k-2}(x)+\frac{1}{2}(k-1)(-1+2 x) S_{k-1}(x)+\frac{1}{4} k(k-1)(-1+2 x) S_{k}(x) .
\end{aligned}
$$

Step 3. After routine coefficient extraction based on (3.15), we obtain

$$
\begin{gathered}
{\left[\frac{x^{n}}{n !}\right] V_{2}^{\text {los }}(x) B(x)=-\frac{1}{4} B_{n+2}+\left(\frac{n}{2}+\frac{1}{4}\right) B_{n+1}+\left(-\frac{n}{2}+\frac{1}{4}\right) B_{n}} \\
{\left[\frac{x^{n}}{n !}\right] V_{2}^{\text {los }}(x) S_{k-2}(x)=\frac{n}{2} S_{n-1, k-2}+\frac{1}{2}(k-1)\left(-S_{n, k-1}+2 n S_{n-1, k-1}\right)} \\
+\frac{1}{4} k(k-1)\left(-S_{n, k}+2 n S_{n-1, k}\right) .
\end{gathered}
$$

It is possible to simplify the expression obtained for $\left[\frac{x^{n}}{n !}\right] V_{2}^{\operatorname{los}}(x) S_{k-2}(x)$ by replacing each occurrence of the left hand sides of identities (3.26) and (3.28) by the corresponding right hand sides. This gives

$$
\left[\frac{x^{n}}{n !}\right] V_{2}^{\text {los }}(x) S_{k-2}(x)=\left(\frac{1}{2} n(k-1)-\frac{1}{4} k(k-1)\right) S_{n, k}+\frac{1}{2}(n+1-k) S_{n, k-1} .
$$

Steps 4 and 5. Division of expression (4.2) by $B_{n}$ gives the exact value $(2.3)$ of $\mu_{n}$, while its asymptotic approximation (2.4) is easily obtained from Lemma 3.3.

Similarly, division of expression (4.3) by $S_{n, k}$ gives the exact value (2.5) of $\mu_{n, k}$, while, after a routine computation based on Lemma 3.2, we obtain the asymptotic approximation

$$
\mu_{n, k}=\frac{1}{2} n(k-1)-\frac{1}{4} k(k-1)+O\left(n\left(1-\frac{1}{k}\right)^{n}\right), \quad n \rightarrow \infty,
$$

which is a refinement of (2.6). This concludes the proof of Theorem 2.2.

\subsection{Some remarks}

There is an "explicit" expression for the generating function of the $q$-Stirling distribution $S(q)$ (see e.g. [16]), namely,

$$
S_{n, k}(q)=\frac{1}{[k]_{q} !} \sum_{j=1}^{k}(-1)^{k-j}\left[\begin{array}{c}
k \\
j
\end{array}\right]_{q} q^{\left(\begin{array}{c}
k-j \\
2
\end{array}\right)}[j]_{q}^{n}
$$

where $\left[\begin{array}{l}n \\ k\end{array}\right]_{q}$ is the usual $q$-binomial coefficient. It seems difficult to recover the exact average values of the $q$-Stirling distributions from the above expression. However, it is 
well-known that "generating functions find averages" (see e.g. [36]). In the case of our $q$-Stirling numbers, it is easy to derive from the recurrence (2.2) that

$$
F_{k}(x, q):=\sum_{n \geq 0} S_{n, k}(q) x^{n}=\frac{q^{\left(\begin{array}{c}
k \\
2
\end{array}\right)} x^{k}}{(1-x)\left(1-[2]_{q} x\right) \cdots\left(1-[k]_{q} x\right)} .
$$

It is possible to recover the results presented in this section from this generating function identity. We can even obtain many more results, such as values of variances and some limit laws (see [19]).

\section{$5 \quad$ Number of linear crossings}

\subsection{Proof of Theorem 2.3}

Computation of $V_{2}^{\mathrm{cr}^{(\ell)}}(x)$. By definition, $V_{2}^{\mathrm{c}^{(\ell)}}(x)$ is the exponential generating function of the sequence $\left(v_{n}\right)_{n \geq 0}$ with $v_{n}:=v_{n, 2}^{\mathrm{cr}(\ell)}=\sum_{\pi \in \Pi_{n}^{2}} \mathrm{cr}(\ell)(\pi)$.

We can interpret $v_{n}$ as the number of pairs $(\pi, C)$, where $\pi$ is a 2-partition of $[n]$ and $C$ is a linear crossing of $\pi$. Such a pair will be called an underlined 2-partition.

An underlined partition $(\pi, C)$ with $C=(i, j)(k, \ell)$ can be identified with the restricted growth function $w(\pi)$ in which the letters $w_{i}, w_{j}, w_{k}$ and $w_{\ell}$ are colored. For instance, there are 3 linear crossings in $\pi=14 / 25 / 367: C_{1}=(1,4)(2,5), C_{2}=(1,4)(3,6)$

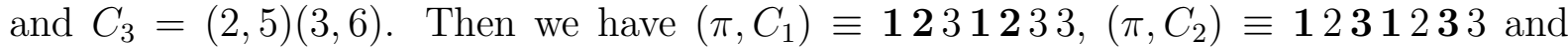
$\left(\pi, C_{3}\right) \equiv 1231233$.

Using this correspondence, it is not hard to see that an underlined 2-partition $(\pi, C)$ can be decomposed uniquely in one of the following two ways:

(a) $(\pi, C) \equiv u_{1} \mathbf{1} 2^{k_{1}} \mathbf{2} 11^{k_{2}} \mathbf{2} u_{2}$,

(b) $(\pi, C) \equiv v_{1} 21^{\ell_{1}} 122^{\ell_{2}} 1 v_{2}$,

with $u_{1}=\epsilon$ or $u_{1} \in 1\{1,2\}^{*}, u_{2} \in\{1,2\}^{*}$ and $k_{1}, k_{2} \geq 0 ; v_{1} \in 1\{1,2\}^{*}, v_{2} \in\{1,2\}^{*}$ and $\ell_{1}, \ell_{2} \geq 0$.

Simple rules of counting and manipulations of generating functions (see e.g. $[13,36]$ ) then show that the ordinary generating functions $F^{(a)}(x)$ and $F^{(b)}(x)$ for underlined 2-partitions of $[n], n \in \mathbb{N}$ admitting a decomposition of type $(a)$ and $(b)$, respectively, are given by

$$
\begin{aligned}
& F^{(a)}(x)=\left(1+x \frac{1}{1-2 x}\right) x\left(\frac{1}{1-x}\right) x^{2}\left(\frac{1}{1-x}\right) x\left(\frac{1}{1-2 x}\right)=\frac{x^{4}(1-x)}{(1-x)^{2}(1-2 x)^{2}} \\
& F^{(b)}(x)=\left(x \frac{1}{1-2 x}\right) x\left(\frac{1}{1-x}\right) x^{2}\left(\frac{1}{1-x}\right) x\left(\frac{1}{1-2 x}\right)=\frac{x^{5}}{(1-x)^{2}(1-2 x)^{2}} .
\end{aligned}
$$

Thus, the number of underlined 2-partitions of $[n]$, i.e., $v_{n}$, is just the coefficient of $x^{n}$ in the power series

$$
F(x)=F^{(a)}(x)+F^{(b)}(x)=\frac{x^{4}}{(1-x)^{2}(1-2 x)^{2}} .
$$




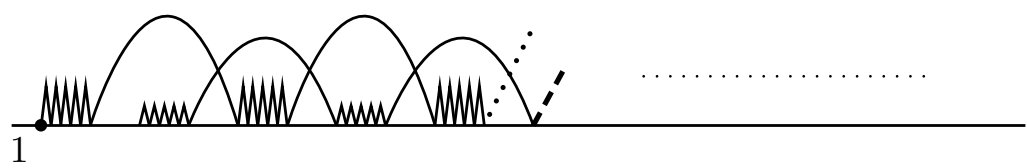

Figure 2: Linear representation of a 2-partition

A routine coefficient extraction based on the partial fraction decomposition

$$
F(x)=\frac{x^{4}}{(1-x)^{2}(1-2 x)^{2}}=x^{4}\left(\frac{1}{(1-x)^{2}}+\frac{4}{1-x}+\frac{4}{(1-2 x)^{2}}-\frac{8}{1-2 x}\right)
$$

gives

$$
v_{n}=\sum_{\pi \in \Pi_{n}^{2}} \mathrm{cr}^{(\ell)}(\pi)= \begin{cases}0, & \text { if } n \leq 3 \\ (n-5) 2^{n-2}+n+1, & \text { if } n \geq 4\end{cases}
$$

A straightforward computation then leads to

$$
V_{2}^{\mathrm{cr}(\ell)}(x)=\sum_{n \geq 0} v_{n} \frac{x^{n}}{n !}=\left(\frac{x}{2}-\frac{5}{4}\right) e^{2 x}+(x+1) e^{x}+\frac{1}{4} .
$$

Of course, there are many other ways to compute the coefficients $v_{n}$. We now present another way based on a decomposition of 2-partitions which is particularly well-adapted to the computation of the coefficients $v_{n}$ in the case of circular crossings.

Via its restricted growth function, a 2-partition can be viewed as a word on the alphabet $\{1,2\}$ whose first letter is 1 . It is then immediate to see that each 2-partition $\pi$ can be decomposed uniquely as

$$
\pi=1^{\ell_{1}} 2^{m_{1}} 1^{\ell_{2}} 2^{m_{2}} \cdots 1^{\ell_{k}} 2^{m_{k}} 1^{\ell_{k+1}}
$$

for some $k \geq 1$, where $\ell_{j}, m_{j} \geq 1$ for $1 \leq j \leq k$, and $\ell_{k+1} \geq 0$.

For $k \geq 1$, denote by $\mathcal{T}_{k}$ the set of 2 -partitions with such a decomposition. A moment's thought (see Figure 2) will convince the reader that

- if $\pi \in \mathcal{T}_{1}, \mathrm{cr}^{(\ell)}(\pi)=0$,

- if $\pi \in \mathcal{T}_{k}, k \geq 2, \mathrm{cr}^{(\ell)}(\pi)= \begin{cases}2 k-3, & \text { if } \ell_{k+1}=0 \\ 2 k-2, & \text { if } \ell_{k+1} \geq 1 .\end{cases}$

It is easy to see from the definition of $\mathcal{T}_{k}$ that the ordinary generating functions $G_{k}^{(a)}(x)$ and $G_{k}^{(b)}(x)$ of 2-partitions of $[n], n \in \mathbb{N}$, in $\mathcal{T}_{k}$ satisfying $\ell_{k+1}=0$ and $\ell_{k+1} \geq 1$, respectively, are given by $G_{k}^{(a)}(x)=\left(\frac{x}{1-x}\right)^{2 k}$ and $G_{k}^{(b)}(x)=\left(\frac{x}{1-x}\right)^{2 k+1}$. It follows that for $k \geq 2$ we have

$$
\begin{aligned}
\sum_{\pi \in \mathcal{T}_{k}} \mathrm{cr}(\pi) x^{\|\pi\|} & =(2 k-3) G_{k}^{(a)}(x)+(2 k-2) G_{k}^{(b)}(x) \\
& =(2 k-3)\left(\frac{x}{1-x}\right)^{2 k}+(2 k-2)\left(\frac{x}{1-x}\right)^{2 k+1}
\end{aligned}
$$


where we have set $\|\pi\|=n$ for $\pi \in \Pi_{n}$.

Using the latter equality and the formal identity $\sum_{k \geq 1} k y^{k}=\frac{y}{(1-y)^{2}}$, after a routine computation we obtain

$$
F(x)=\sum_{n \geq 0} v_{n} x^{n}=\sum_{k \geq 2} \sum_{\pi \in \mathcal{T}_{k}} \mathrm{c} r^{(\ell)}(\pi) x^{\|\pi\|}=\frac{x^{4}}{(1-x)^{2}(1-2 x)^{2}},
$$

as it was previously derived.

Since $V_{2}^{\text {cr }}(x)(x)$ see $(5.1)$ ) can be written in the form (3.9), we can continue the process described in Section 3.2.

Step 2. Using (3.10), (3.11) and (3.12), we can write

$$
\begin{aligned}
V_{2}^{c r^{(\ell)}}(x) B(x) & =\frac{1}{4} B(x)+\frac{1}{4}(9+2 x) B^{\prime}(x)+\frac{1}{4}(-5+2 x) B^{\prime \prime}(x) \\
V_{2}^{c r^{(\ell)}}(x) S_{k-2}(x) & =\frac{3 x}{2} S_{k-2}(x)+\frac{1}{2}(k-1)(-3+4 x) S_{k-1}(x)+\frac{1}{4} k(k-1)(-5+2 x) S_{k}(x) .
\end{aligned}
$$

Step 3. After routine coefficient extraction based on (3.15), we obtain

$$
\begin{gathered}
{\left[\frac{x^{n}}{n !}\right] V_{2}^{\mathrm{cr}^{(\ell)}}(x) B(x)=-\frac{5}{4} B_{n+2}+\left(\frac{n}{2}+\frac{9}{4}\right) B_{n+1}+\left(\frac{n}{2}+\frac{1}{4}\right) B_{n}} \\
{\left[\frac{x^{n}}{n !}\right] V_{2}^{\mathrm{cr}(\ell)}(x) S_{k-2}(x)=\frac{3 n}{2} S_{n-1, k-2}+\frac{1}{2}(k-1)\left(-3 S_{n, k-1}+4 n S_{n-1, k-1}\right)} \\
+\frac{1}{4} k(k-1)\left(-5 S_{n, k}+2 n S_{n-1, k}\right) .
\end{gathered}
$$

It is possible to simplify the expression obtained for $\left[\frac{x^{n}}{n !}\right] V_{2}^{\text {cr }}{ }^{(\ell)}(x) S_{k-2}(x)$ by replacing each occurrence of the left hand sides of identities (3.26) and (3.28) by the corresponding right hand sides. This gives

$$
\left[\frac{x^{n}}{n !}\right] V_{2}^{\mathrm{cr}(\ell)}(x) S_{k-2}(x)=\left(\frac{1}{2} n(k-1)-\frac{5}{4} k(k-1)\right) S_{n, k}+\frac{3}{2}(n+1-k) S_{n, k-1} .
$$

Steps 4 and 5. Division of expression (5.2) by $B_{n}$ gives the exact value $(2.7)$ of $\mu_{n}$, while its asymptotic approximation (2.8) is easily obtained from Lemma 3.3.

Similarly, division of expression (5.3) by $S_{n, k}$ gives the exact value (2.9) of $\mu_{n, k}$, while, after a routine computation based on Lemma 3.2, we get the asymptotic approximation

$$
\mu_{n, k}=\frac{1}{2} n(k-1)-\frac{5}{2}\left(\begin{array}{l}
k \\
2
\end{array}\right)+O\left(n\left(1-\frac{1}{k}\right)^{n}\right), \quad n \rightarrow \infty,
$$

which is a refinement of (2.10). This concludes the proof of Theorem 2.3. 


\subsection{Some Remarks}

It is worth noting that there has been considerable interest in studying crossings in matchings (set partitions each block of which has exactly two elements) and set partitions.

In the case of the number of crossings in matchings, a remarkable formula, often called the Touchard-Riordan formula, was made explicit by Riordan [28], who also mentioned the exact values of the average and the variance of the number of crossings in matchings. Though they are "contained" in the Touchard-Riordan formula, it is not a priori clear whether they admit a simple form. Later, Flajolet and Noy [11] used a certain decomposition of the number of crossings to give a "direct" proof for the average value. This decomposition was later generalized by the author [20] to compute the average number of linear crossings in a random set partition and is at the origin of this work.

Let $T_{n, k}(q)$ be the generating function of set partitions of $[n]$ into $k$ blocks with respect to the number of linear crossings, i.e., $T_{n, k}(q)=\sum_{\pi \in \Pi_{n}^{k}} q^{r^{(\ell)}(\pi)}$. Biane [1] (see also [22]) found a continued fraction expansion for the generating function $\sum_{n \geq k \geq 0} T_{n, k}(q) a^{k} t^{n}$. Recently, Stanton, Zeng and the author (see Equation 28 in [21]) proved that

$$
\sum_{n \geq k \geq 0} T_{n, k}(q) a^{k} t^{n}=\sum_{k=0}^{\infty} \frac{(a q t)^{k}}{\prod_{i=1}^{k}\left(q^{i}-q^{i}[i]_{q} t+a(1-q)[i]_{q} t\right)}
$$

from which they derive the following remarkable formula for $T_{n, k}(q)$ (see Equation 32 in $[21])$ :

$$
T_{n, k}(q)=\sum_{j=1}^{k}(-1)^{k-j} \frac{[j]_{q}^{n}}{[j]_{q} !} \sum_{i=0}^{k-j} \frac{(1-q)^{i}}{[k-j-i]_{q} !} q^{\left(\begin{array}{c}
k-j-i+1 \\
2
\end{array}\right)-k j}\left(\left(\begin{array}{c}
n \\
i
\end{array}\right) q^{j}+\left(\begin{array}{c}
n \\
i-1
\end{array}\right)\right) .
$$

Another remarkable formula for $T_{n, k}(q)$ was established recently by Josuat-Vergès and Rubey [18]:

$$
T_{n, k}(q)=\frac{1}{(1-q)^{n-k}} \sum_{j=0}^{k} \sum_{i=j}^{n-k}(-1)^{i}\left(\left(\begin{array}{c}
n \\
k+i
\end{array}\right)\left(\begin{array}{c}
n \\
k-j
\end{array}\right)-\left(\begin{array}{c}
n \\
k+i+1
\end{array}\right)\left(\begin{array}{c}
n \\
k-j-1
\end{array}\right)\right)\left[\begin{array}{c}
i \\
j
\end{array}\right]_{q} q^{\left(\begin{array}{c}
j+1 \\
2
\end{array}\right)} .
$$

Again, though the average number of linear crossings is "contained" in the above two formulas, it is not a priori clear whether they admit a simple form. However, it is possible to recover the exact average values presented in this section and even exact values for the variance of the number of linear crossings (see [19]) from the generating function (5.4). Moreover, a limit law will be given in [19].

Finally, we want to point out that there exist combinatorial parameters which have the same distribution (hence, the same average value) as the number of linear crossings

on each $\Pi_{n}^{k}$. This is the case of the number of nestings of two arcs [22] and the major index for set partitions introduced in [5]. 


\section{$6 \quad$ Number of circular crossings}

\subsection{Proof of Theorem 2.4}

Computation of $V_{2}^{\mathrm{cr}^{(c)}}(x)$. By definition, $V_{2}^{\mathrm{cr}^{(c)}}(x)$ is the exponential generating function of the sequence $\left(v_{n}\right)_{n \geq 0}$ with $v_{n}:=v_{n, 2}^{c r^{(c)}}=\sum_{\pi \in \Pi_{n}^{2}} \mathrm{cr}\left({ }^{(c)}(\pi)\right.$.

Suppose we are given a 2-partition $\pi$ in $\mathcal{T}_{k}$. By definition of $\mathcal{T}_{k}$ (see Section 5.1), this means that there exist integers $\ell_{j}, m_{j} \geq 1$ for $1 \leq j \leq k$, and $\ell_{k+1} \geq 0$ such that

$$
\pi=1^{\ell_{1}} 2^{m_{1}} 1^{\ell_{2}} 2^{m_{2}} \cdots 1^{\ell_{k}} 2^{m_{k}} 1^{\ell_{k+1}} .
$$

A moment's thought (see Figure 3) will convince the reader that:

- if $k=1, \mathrm{cr}^{(c)}(\pi)=0$,

- if $k \geq 3, \mathrm{cr}^{(c)}(\pi)=2 k$.
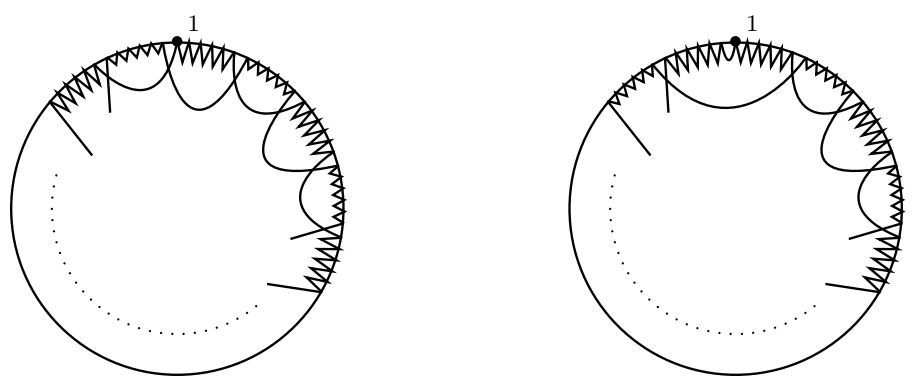

Figure 3: Circular representation of a 2-partition

This implies that, for $k \geq 3$, we have

$$
\sum_{\pi \in \mathcal{T}_{k}} \mathrm{cr}(c)(\pi) x^{\|\pi\|}=2 k\left(\frac{x}{1-x}\right)^{2 k} \frac{1}{1-x}=2 k \frac{x^{2 k}}{(1-x)^{2 k+1}},
$$

since the generating function $G_{k}(x)=\sum_{\pi \in \mathcal{T}_{k}} x^{\|\pi\|}$ of 2-partitions in $\mathcal{T}_{k}$ is given by $G_{k}(x)=$ $\left(\frac{x}{1-x}\right)^{2 k}\left(\frac{1}{1-x}\right)$. Using the formal identity $\sum_{k \geq 0} k y^{k}=\frac{y}{(1-y)^{2}}$, it is then easy to obtain the identity

$$
\sum_{k \geq 3} \sum_{\pi \in \mathcal{T}_{k}} c r^{(c)}(\pi) x^{\|\pi\|}=\sum_{k \geq 3} 2 k \frac{x^{2 k}}{(1-x)^{2 k+1}}=\frac{2 x^{6}\left(3-6 x+x^{2}\right)}{(1-2 x)^{2}(1-x)^{5}} .
$$

For the case $k=2$, we have to be more careful. By definition, a partition in $\mathcal{T}_{2}$ can be written in the form $\pi=1^{\ell_{1}} 2^{m_{1}} 1^{\ell_{2}} 2^{m_{2}} 1^{\ell_{3}}$, with $\ell_{1}, \ell_{2}, m_{1}, m_{2} \geq 1$ and $\ell_{3} \geq 0$. We distinguish four cases. Instead of long-winded explanations, we prefer to present the results in the table given in Figure 4. The first column lists the different cases considered, while the second column indicates the corresponding circular representations. In each case, 


\begin{tabular}{|c|c|c|c|}
\hline & $\begin{array}{l}\text { sketch of the circular } \\
\text { representation }\end{array}$ & $\begin{array}{l}\text { value } \\
\text { of } \mathrm{cr} r^{(c)}\end{array}$ & $\begin{array}{l}\text { generating } \\
\text { function }\end{array}$ \\
\hline $\begin{array}{l}\quad \text { case (a) } \\
\ell_{1}=\ell_{2}=1, \ell_{3}=0 \\
m_{1}=m_{2}=1\end{array}$ & & 1 & $x^{4}$ \\
\hline $\begin{array}{l}\quad \text { case (b) } \\
\ell_{1}=\ell_{2}=1, \ell_{3}=0 \\
m_{1}+m_{2} \geq 3\end{array}$ & & 2 & $x^{2}\left(\left(\frac{x}{1-x}\right)^{2}-x^{2}\right)$ \\
\hline $\begin{array}{l}\quad \text { case }(\mathrm{c}) \\
\ell_{1}+\ell_{2}+\ell_{3} \geq 3 \\
m_{1}=m_{2}=1\end{array}$ & & 2 & $x^{2}\left(\frac{x}{1-x}\right)^{2} \frac{1}{1-x}$ \\
\hline $\begin{array}{l}\quad \text { case }(\mathrm{d}) \\
\ell_{1}+\ell_{2}+\ell_{3} \geq 3 \\
m_{1}+m_{2} \geq 3\end{array}$ & ${ }_{p}$ & 4 & $\left(\frac{x}{1-x}\right)^{2} \frac{1}{1-x}\left(\left(\frac{x}{1-x}\right)^{2}-x^{2}\right)$ \\
\hline
\end{tabular}

Figure 4: Circular crossings in a 2-partition in $\mathcal{T}_{2}$.

the statistic $\mathrm{cr}^{(c)}$ assumes a constant value: the third column provides these constants. Finally, the (ordinary) generating function of 2-partitions in each case is given in the fourth column. For instance, the first and third row read as follows:

- case (a): there is one and only one partition $\pi=13 / 24, \mathrm{cr}^{(c)}(\pi)=1$ and the generating function for this case is $x^{4}$.

- case (c): the parameter $\mathrm{cr}^{(c)}$ is equal to 2 and the generating function of this class is $x^{2}\left(\frac{x}{1-x}\right)^{2} \frac{1}{1-x}$. The other rows have to be read similarly.

Let $F_{1}, F_{2}, F_{3}$ and $F_{4}$ be the generating functions of 2-partitions in case $(a),(b),(c)$ and 
$(d)$, respectively. It follows from a reading of the entire table that

$$
\sum_{\pi \in \mathcal{T}_{2}} c r^{(c)}(\pi) x^{\|\pi\|}=1 F_{1}(x)+2 F_{2}(x)+2 F_{3}(x)+4 F_{4}(x)=x^{4}+\frac{2 x^{5}\left(5-4 x+x^{2}\right)}{(1-x)^{5}} .
$$

Combining (6.1) and the latter equality, we obtain

$$
\begin{aligned}
\sum_{\pi \in \Pi^{2}} c r^{(c)}(\pi) x^{\|\pi\|} & =\sum_{k \geq 1} \sum_{\pi \in \mathcal{T}_{k}} c r^{(c)}(\pi) x^{\|\pi\|}=\sum_{\pi \in \mathcal{T}_{2}} c r^{(c)}(\pi) x^{\|\pi\|}+\sum_{k \geq 3} \sum_{\pi \in \mathcal{T}_{k}} c r^{(c)}(\pi) x^{\|\pi\|} \\
& =x^{4}+\frac{2 x^{5}\left(5-4 x+x^{2}\right)}{(1-x)^{5}}+\frac{2 x^{6}\left(3-6 x+x^{2}\right)}{(1-2 x)^{2}(1-x)^{5}} \\
& =x^{4}+\frac{2 x^{5}\left(5-11 x+4 x^{2}\right)}{(1-x)^{3}(1-2 x)^{2}}
\end{aligned}
$$

A routine coefficient extraction based on the partial fraction decomposition

$$
\begin{aligned}
x^{4}+\frac{2 x^{5}\left(5-11 x+4 x^{2}\right)}{(1-x)^{3}(1-2 x)^{2}}=- & \frac{5 x}{2}-2 x^{2}+x^{4}-\frac{4}{(1-x)^{3}}+\frac{10}{(1-x)^{2}}-\frac{6}{1-x} \\
& +\frac{1}{4(1-2 x)^{2}}-\frac{1}{4(1-2 x)}
\end{aligned}
$$

gives

$$
v_{n}=\sum_{\pi \in \Pi_{n}^{2}} c r^{(c)}(\pi)= \begin{cases}0, & \text { if } 0 \leq n \leq 3 \\ 1, & \text { if } n=4 ; \\ n 2^{n-2}+4 n-2 n^{2}, & \text { if } n \geq 5\end{cases}
$$

A straightforward computation leads to

$$
V_{2}^{\text {cr }}{ }^{(c)}(x)=\frac{1}{2} x e^{2 x}+2 x(-x+1) e^{x}+\frac{x^{4}}{24}-x^{2}-\frac{5}{2} x .
$$

Since $V_{2}^{\text {cr }}{ }^{(c)}(x)$ can be written in the form (3.9), we can continue the process described in Section 3.2.

Step 2. Using (3.10), (3.11) and (3.12), we can write

$$
\begin{aligned}
V_{2}^{\text {cr(c) }}(x) B(x)= & \frac{1}{24}\left(x^{4}-24 x^{2}-60 x\right) B(x)+\frac{1}{2}\left(-4 x^{2}+3 x\right) B^{\prime}(x)+\frac{x}{2} B^{\prime \prime}(x) \\
V_{2}^{\text {stat }}(x) S_{k-2}(x)= & \frac{1}{24}\left(x^{4}-72 x^{2}\right) S_{k-2}(x)+(k-1)\left(-2 x^{2}+3 x\right) S_{k-1}(x) \\
& +\frac{1}{2} k(k-1) x S_{k}(x) .
\end{aligned}
$$


Step 3. After routine coefficient extraction based on (3.15), we obtain

$$
\begin{aligned}
{\left[\frac{x^{n}}{n !}\right] V_{2}^{\mathrm{cr}(c)}(x) B(x)=} & \frac{n}{2} B_{n+1}+\frac{3 n}{2} B_{n}-2 n\left(n+\frac{1}{4}\right) B_{n-1}-(n)_{2} B_{n-2}+\frac{1}{24}(n)_{4} B_{n-4} \\
{\left[\frac{x^{n}}{n !}\right] V_{2}^{\mathrm{cr}(c)}(x) S_{k-2}(x)=\frac{1}{24} } & \left((n)_{4} S_{n-4, k-2}-72(n)_{2} S_{n-2, k-2}\right) \\
& +(k-1)\left(-2(n)_{2} S_{n-2, k-1}+3 n S_{n-1, k-1}\right) \\
& +\frac{1}{2} k(k-1) n S_{n-1, k} .
\end{aligned}
$$

It is possible to simplify the expression obtained for $\left[\frac{x^{n}}{n !}\right] V_{2}^{\text {cr }}{ }^{(c)}(x) S_{k-2}(x)$ by replacing each occurrence of the left hand sides of identities (3.28) and

$$
S_{n-2, k-1}=\frac{1}{k-1}\left(S_{n-1, k-1}-S_{n-2, k-2}\right)
$$

by the corresponding right hand sides. This gives

$$
\begin{gathered}
{\left[\begin{array}{l}
x^{n} \\
n !
\end{array}\right] V_{2}^{c^{(c)}}(x) S_{k-2}(x)=\frac{n(k-1)}{2} S_{n, k}+\frac{n}{2}(-4 n-1+5 k) S_{n-1, k-1}} \\
-(n)_{2} S_{n-2, k-2}+\frac{(n)_{4}}{24} S_{n-4, k-2} .
\end{gathered}
$$

Steps 4 and 5. Division of expression (6.3) by $B_{n}$ gives the exact value (2.11) of $\mu_{n}$, while its asymptotic approximation (2.12) is easily obtained from Lemma 3.3.

Similarly, division of expression (6.5) by $S_{n, k}$ gives the exact value (2.13) of $\mu_{n, k}$, while, after a routine computation based on Lemma 3.2, we get the asymptotic approximation

$$
\mu_{n, k}=\frac{1}{2} n(k-1)+O\left(n^{2}\left(1-\frac{1}{k}\right)^{n}\right), \quad n \rightarrow \infty,
$$

which is a refinement of (2.14). This concludes the proof of Theorem 2.4.

\subsection{Some Remarks}

While the combinatorial parameter $\mathrm{cr}^{(\ell)}$ has received a considerable interest, it seems that the natural parameter $\mathrm{cr}\left({ }^{(c)}\right.$ was ignored. It is interesting to ask whether this parameter admits "closed" formulas similar to the formulas for $\mathrm{cr}\left({ }^{(\ell)}\right.$ presented in the previous section. Some results on the asymptotic distribution of the parameter $\mathrm{cr}{ }^{(c)}$ will be given in [19].

\section{Number of overlappings}

\subsection{Proof of Theorem 2.5}

Computation of $V_{2}^{\mathrm{ovl}}(x)$. By definition, $V_{2}^{\mathrm{ovl}}(x)$ is the exponential generating function of the sequence $\left(v_{n}\right)_{n \geq 0}$ with $v_{n}:=v_{n, 2}^{\text {ovl }}=\sum_{\pi \in \Pi_{n}^{2}} \mathrm{O} v l(\pi)$. 
Since ovl assumes only the values 0 or 1 on $\Pi_{n}^{2}$, the term $v_{n}$ is just the number of 2partitions of $[n]$ that overlap, i.e., the number of 2-partitions $\pi=B_{1} / B_{2}$ of $[n]$ such that $1=\min B_{1}<\min B_{2}<\max B_{1}<\max B_{2}=n$. It is easy to see that there are exactly $2^{n-2}$ partitions $\pi=B_{1} / B_{2}$ of $[n]$ such that $\min B_{1}=1$ and $\max B_{2}=n$, and among them only $n-1$ do not overlap (these are the partitions $\pi=1^{k} 2^{n-k}$ with $1 \leq k \leq n-1$ ). Consequently

$$
v_{n}=\sum_{\pi \in \Pi_{n}^{2}} \mathrm{ovl}(\pi)= \begin{cases}2^{n-2}-n+1, & \text { if } n \geq 2 \\ 0, & \text { if } n \leq 1\end{cases}
$$

A straightforward computation leads to

$$
V_{2}^{\mathrm{o} v l}(x)=\sum_{n \geq 0} v_{n} \frac{x^{n}}{n !}=\frac{1}{4} e^{2 x}+(-x+1) e^{x}-\frac{x}{2}-\frac{5}{4} .
$$

Since $V_{2}^{\text {ovl }}(x)$ can be written in the form (3.9), we can continue the process described in Section 3.2.

Step 2. Using (3.10), (3.11) and (3.12), we can write

$$
\begin{aligned}
V_{2}^{\mathrm{o} v l}(x) B(x) & =\left(-\frac{x}{2}-\frac{5}{4}\right) B(x)+\left(-x+\frac{3}{4}\right) B^{\prime}(x)+\frac{1}{4} B^{\prime \prime}(x) \\
V_{2}^{\mathrm{o} v l}(x) S_{k-2}(x) & =-\frac{3 x}{2} S_{k-2}(x)-(k-1)\left(x-\frac{3}{2}\right) S_{k-1}(x)+\frac{1}{4} k(k-1) S_{k}(x) .
\end{aligned}
$$

Step 3. After routine coefficient extraction based on (3.15), we obtain

$$
\begin{gathered}
{\left[\frac{x^{n}}{n !}\right] V_{2}^{\mathrm{o} v l}(x) B(x)=\frac{1}{4} B_{n+2}+\frac{3}{4} B_{n+1}-\left(n+\frac{5}{4}\right) B_{n}-\frac{n}{2} B_{n-1}} \\
{\left[\frac{x^{n}}{n !}\right] V_{2}^{\mathrm{ovl}}(x) S_{k-2}(x)=-\frac{3 n}{2} S_{n-1, k-2}-\frac{1}{2}(k-1)\left(2 n S_{n-1, k-1}-3 S_{n, k-1}\right)} \\
+\frac{1}{4} k(k-1) S_{n, k} .
\end{gathered}
$$

It is possible to simplify the expression obtained for $\left[\frac{x^{n}}{n !}\right] V_{2}^{\text {ovl }}(x) S_{k-2}(x)$ by replacing each occurrence of the left hand side of identity (3.26) by the corresponding right hand side. This gives

$$
\begin{gathered}
{\left[\frac{x^{n}}{n !}\right] V_{2}^{\mathrm{o} v l}(x) S_{k-2}(x)=\frac{1}{4}} \\
k(k-1) S_{n, k}-\frac{3}{2}(n+1-k) S_{n, k-1} \\
+\frac{1}{2} n(k-1) S_{n-1, k-1} .
\end{gathered}
$$

Steps 4 and 5. Division of expression (7.2) by $B_{n}$ gives the exact value $(2.15)$ of $\mu_{n}$, while its asymptotic approximation (2.16) is easily obtained from Lemma 3.3. 
Similarly, division of expression (7.3) by $S_{n, k}$ gives the exact value (2.17) of $\mu_{n, k}$, while, after a routine computation based on Lemma 3.2, we obtain the asymptotic approximation

$$
\mu_{n, k}=\frac{1}{2}\left(\begin{array}{l}
k \\
2
\end{array}\right)+O\left(n\left(1-\frac{1}{k}\right)^{n}\right), \quad n \rightarrow \infty,
$$

which is a refinement of (2.18). This concludes the proof of Theorem 2.5.

\subsection{Some remarks}

Although it seems that the parameter number of overlappings was never considered before, it is naturally suggested by the article [12], in which Flajolet and Schott considered non-overlappings partitions. In particular, they obtained the generating function of nonoverlapping partitions in the form of a continued fraction expansion. It is worth noting that a continued fraction expansion for the generating function of set partitions with respect to the number of overlappings follows painlessly from earlier combinatorial investigations (see e.g. $[10,12])$.

We also want to point out that, as $n \rightarrow \infty, \mu_{n, k}$ converges to $\left(\begin{array}{l}k \\ 2\end{array}\right)$. That this is also the average number of inversions in a random permutation of size $k$ is far from being a coincidence, as we will explain in future work.

\section{Number of Embracings}

\subsection{Proof of Theorem 2.6}

Computation of $V_{2}^{\mathrm{e} m b}(x)$. By definition, $V_{2}^{\mathrm{e} m b}(x)$ is the exponential generating function of the sequence $\left(v_{n}\right)_{n \geq 0}$ with $v_{n}:=v_{n, 2}^{\mathrm{e} m b}=\sum_{\pi \in \Pi_{n}^{2}} \mathrm{e} m b(\pi)$.

Since emb assumes only the values 0 or 1 on $\Pi_{n}^{2}$, the term $v_{n}$ is just the number of 2-partitions of $[n]$ which are embracing, i.e., the number of 2-partitions $\pi=B_{1} / B_{2}$ of $[n]$ such that $1=\min B_{1}<\min B_{2} \leq \max B_{2}<\max B_{1}=n$, or, equivalently, such that $\min B_{1}=1$ and $\max B_{1}=n$. Obviously, for $n \geq 2$, there are exactly $2^{n-2}-1$ such 2-partitions. Consequently

$$
v_{n}=\sum_{\pi \in \Pi_{n}^{2}} \operatorname{emb}(\pi)= \begin{cases}2^{n-2}-1, & \text { if } n \geq 2 \\ 0, & \text { if } n \leq 1\end{cases}
$$

A straightforward computation leads to

$$
V_{2}^{\mathrm{e} m b}(x)=\sum_{n \geq 0} v_{n} \frac{x^{n}}{n !}=\frac{1}{4} e^{2 x}-e^{x}+\frac{x}{2}+\frac{3}{4} .
$$

Since $V_{2}^{\mathrm{e} m b}(x)$ can be written in the form (3.9), we can continue the process described in Section 3.2. 
Step 2. Using (3.10), (3.11) and (3.12), we can write

$$
\begin{aligned}
V_{2}^{\mathrm{e} m b}(x) B(x) & =\left(\frac{x}{2}+\frac{3}{4}\right) B(x)-\frac{5}{4} B^{\prime}(x)+\frac{1}{4} B^{\prime \prime}(x) \\
V_{2}^{\mathrm{e} m b}(x) S_{k-2}(x) & =\frac{x}{2} S_{k-2}(x)-\frac{1}{2}(k-1) S_{k-1}(x)+\frac{1}{4} k(k-1) S_{k}(x) .
\end{aligned}
$$

Step 3. After routine coefficient extraction based on (3.15), we obtain

$$
\begin{aligned}
{\left[\frac{x^{n}}{n !}\right] V_{2}^{\mathrm{e} m b}(x) B(x) } & =\frac{1}{4} B_{n+2}-\frac{5}{4} B_{n+1}+\frac{3}{4} B_{n}+\frac{n}{2} B_{n-1} \\
{\left[\frac{x^{n}}{n !}\right] V_{2}^{\mathrm{e} m b}(x) S_{k-2}(x) } & =\frac{n}{2} S_{n-1, k-2}-\frac{1}{2}(k-1) S_{n, k-1}+\frac{1}{4} k(k-1) S_{n, k} .
\end{aligned}
$$

Steps 4 and 5. Division of expression (8.2) by $B_{n}$ gives the exact value (2.19) of $\mu_{n}$, while its asymptotic approximation (2.20) is easily obtained from Lemma 3.3.

Similarly, division of expression (8.3) by $S_{n, k}$ gives the exact value (2.21) of $\mu_{n, k}$, while, after a routine computation based on Lemma 3.2, we get the asymptotic approximation

$$
\mu_{n, k}=\frac{1}{2}\left(\begin{array}{l}
k \\
2
\end{array}\right)+O\left(\left(1-\frac{1}{k}\right)^{n}\right), \quad n \rightarrow \infty,
$$

which is a refinement of (2.22). This concludes the proof of Theorem 2.6.

\subsection{Some remarks}

It is worth noting that, like for overlappings, it is painless to derive a continued fraction form for the generating function of set partitions with respect to the number of embracings by making use of the combinatorial theory of continued fractions [10].

One can also consider a variation of embracings. Say that two sets $B$ and $B^{\prime}$ strongly embrace each other if, with the implied order structure, $\min (B)<\min \left(B^{\prime}\right)<\max \left(B^{\prime}\right)<$ $\max (B)$. Then one can show that the statistic 'number of strong embracings' has the same distribution (hence, the same average value) on each $\Pi_{n}^{k}$ as the number of overlappings. This can be proved using the combinatorial theory of continued fractions or by a direct combinatorial argument.

\section{Number of occurrences of a 2-Pattern}

\subsection{Proof of Theorem 2.7 and Theorem 2.8}

Computation of $V_{2}^{\text {occ }_{\sigma}}(x)$. Let $\sigma=\sigma_{1} \sigma_{2} \ldots \sigma_{r}$ be a 2-pattern of length $r$. By definition, $V_{2}^{\text {occ }_{\sigma}}(x)$ is the exponential generating function of the sequence $\left(v_{n}\right)_{n \geq 0}$ with $v_{n}:=v_{n, 2}^{\mathrm{occ}_{\sigma}}=\sum_{\pi \in \Pi_{n}^{2}} \mathrm{occ}_{\sigma}(\pi)$.

We can interpret $v_{n}$ as the number of pairs $(\pi, t)$, where $\pi$ is a 2-partition of $[n]$ and $t=\left(i_{1}, i_{2}, \ldots, i_{r}\right)$ is an occurrence of $\sigma$ in $\pi$. Such a pair will be called an underlined 
2-partition. An underlined 2-partition $(\pi, t)$ with $t=\left(i_{1}, i_{2}, \ldots, i_{r}\right)$ can be identified with the restricted growth function $w(\pi)$ in which the letters $w_{i_{1}}, w_{i_{2}}, \ldots, w_{i_{r}}$ are colored. For instance, if $\sigma=212$, the 3 -tuple $t=(2,4,5)$ is an occurrence of $\sigma$ in $\pi=14 / 235$ and $(\pi, t) \equiv 12212$.

Using this correspondence, it is not hard to see that an underlined 2-partition can be decomposed uniquely as

$$
\pi=u_{1} \sigma_{\mathbf{1}} u_{2} \sigma_{\mathbf{2}} \cdots u_{r} \sigma_{\mathbf{r}} u_{r+1} \quad \text { if } \sigma_{1}=1,
$$

with $u_{1} \in\{\epsilon\} \cup 1\{1,2\}^{*}$ and $u_{i} \in\{1,2\}^{*}$ for $i=2, \ldots, r+1$;

$$
\pi=1 v_{1} \sigma_{\mathbf{1}} v_{2} \sigma_{\mathbf{2}} \cdots v_{r} \sigma_{\mathbf{r}} v_{r+1} \quad \text { if } \sigma_{1}=2,
$$

with $v_{i} \in\{1,2\}^{*}$ for $i=1, \ldots, r+1$. In particular, this proves that $v_{n}$ (and thus $V_{2}^{\text {occ }_{\sigma}}(x)$ ) depends only on the first letter of $\sigma$. For $i \in\{1,2\}$, let $v_{n}^{\left(\sigma_{1}=i\right)}$ equal the value of $v_{n}$ when $\sigma_{1}=i$. It follows from the above decomposition of underlined 2-partitions combined with an elementary counting that for $n \geq r$,

$$
v_{n}=\sum_{\pi \in \Pi_{n}^{2}} \operatorname{occ}_{\sigma}(\pi)= \begin{cases}v_{n}^{\left(\sigma_{1}=1\right)}=\left(\begin{array}{c}
n-1 \\
r-1
\end{array}\right) 2^{n-r}+\left(\begin{array}{c}
n-1 \\
r
\end{array}\right) 2^{n-r-1}, & \text { if } \sigma_{1}=1 \\
v_{n}^{\left(\sigma_{1}=2\right)}=\left(\begin{array}{c}
n-1 \\
r
\end{array}\right) 2^{n-r-1}, & \text { if } \sigma_{1}=2 .\end{cases}
$$

To see the above result in the case $\sigma_{1}=2$, for instance, just observe that there are $\left(\begin{array}{c}n-1 \\ r\end{array}\right)$ choices for the positions of the underlined elements $\sigma_{1}, \sigma_{2}, \ldots, \sigma_{\mathrm{r}}$ and then $2^{n-r-1}$ choices for the word $v_{1} v_{2} \cdots v_{r+1}$. The case $\sigma_{1}=1$ can be treated in a similar way.

For $i \in\{1,2\}$, let $V_{2}^{\left(\sigma_{1}=i\right)}(x)$ equal $V_{2}^{\text {occ }_{\sigma}}(x)$ in the case where $\sigma_{1}=i$, i.e., $V_{2}^{\left(\sigma_{1}=i\right)}(x)=$ $\sum_{n \geq 0} v_{n}^{\left(\sigma_{1}=i\right)} \frac{x^{n}}{n !}$. For later simplifications, it is important to note that it suffices to determine only one of the $V_{2}^{\left(\sigma_{1}=i\right)}(x)$ 's to get the other. Indeed, an elementary computation yields

$$
v_{n}^{\left(\sigma_{1}=1\right)}+v_{n}^{\left(\sigma_{1}=2\right)}=\left(\left(\begin{array}{c}
n-1 \\
r-1
\end{array}\right) 2^{n-r}+\left(\begin{array}{c}
n-1 \\
r
\end{array}\right)\right) 2^{n-r-1}+\left(\begin{array}{c}
n-1 \\
r
\end{array}\right) 2^{n-r-1}=\left(\begin{array}{c}
n \\
r
\end{array}\right) 2^{n-r},
$$

from which we deduce easily (using (9.2), for instance) that

$$
V_{2}^{\left(\sigma_{1}=1\right)}(x)+V_{2}^{\left(\sigma_{1}=2\right)}(x)=\sum_{n \geq 0}\left(v_{n}^{\left(\sigma_{1}=1\right)}+v_{n}^{\left(\sigma_{1}=2\right)}\right) \frac{x^{n}}{n !}=\sum_{n \geq 0}\left(\begin{array}{l}
n \\
r
\end{array}\right) 2^{n-r} \frac{x^{n}}{n !}=\frac{x^{r}}{r !} e^{2 x} .
$$

We choose to deal with $V_{2}^{\left(\sigma_{1}=2\right)}(x)$. In order to obtain a convenient expression for $V_{2}^{\left(\sigma_{1}=2\right)}(x)$, we need some additional materials. Let $k$ be a nonnegative integer and $(n)_{k}:=n(n-1)(n-2) \cdots(n-k+1)$, as before. Given a formal power series $A(x):=$ $\sum_{n \geq 0} a_{n} \frac{x^{n}}{n !}$, it is easy to establish the formal identity

$$
\sum_{n \geq 0}(n)_{k} a_{n} \frac{x^{n}}{n !}=x^{k} A^{(k)}(x)
$$


What about the power series $\sum_{n \geq 0}(n-1)_{k} a_{n} \frac{x^{n}}{n !}$ ? Using the Chu-Vandermonde convolution, we have

$$
(n-1)_{k}=\sum_{j=0}^{k}\left(\begin{array}{l}
k \\
j
\end{array}\right)(n)_{j}(-1)_{k-j}=\sum_{j=0}^{k}(-1)^{k-j} \frac{k !}{j !}(n)_{j}
$$

from which we deduce that

$$
\sum_{n \geq 0}(n-1)_{k} a_{n} \frac{x^{n}}{n !}=\sum_{j=0}^{k}(-1)^{k-j} \frac{k !}{j !} x^{j} A^{(j)}(x) .
$$

In particular, for $a_{n}=2^{n}$, we obtain

$$
\sum_{n \geq 0}(n-1)_{k} 2^{n} \frac{x^{n}}{n !}=\sum_{j=0}^{k}(-1)^{k-j} \frac{k !}{j !} x^{j} 2^{j} e^{2 x}=\left(\sum_{j=0}^{k}(-1)^{k-j} \frac{k !}{j !} x^{j} 2^{j}\right) e^{2 x}
$$

We can now express the power series $V_{2}^{\left(\sigma_{1}=2\right)}(x)$ (and $\left.V_{2}^{\left(\sigma_{1}=1\right)}(x)\right)$ in a convenient form. We have seen earlier that $v_{n}^{\left(\sigma_{1}=2\right)}=\left(\begin{array}{c}n-1 \\ r\end{array}\right) 2^{n-r-1}$ for $n \geq r\left(v_{n}^{\left(\sigma_{1}=2\right)}=0\right.$, otherwise $)$. After elementary manipulations, one gets

$$
V_{2}^{\left(\sigma_{1}=2\right)}(x)=\sum_{n \geq r}\left(\begin{array}{c}
n-1 \\
r
\end{array}\right) 2^{n-r-1} \frac{x^{n}}{n !}=\frac{(-1)^{r+1}}{2^{r+1}}+\frac{1}{r ! 2^{r+1}} \sum_{n \geq 0}(n-1)_{r} 2^{n} \frac{x^{n}}{n !}
$$

For any integer $r \geq 0$, set

$$
P_{r}(x):=\sum_{j=0}^{r} \frac{(-1)^{r-j}}{j ! 2^{r+1-j}} x^{j}
$$

Combining (9.5) and (9.4), we arrive at

$$
V_{2}^{\left(\sigma_{1}=2\right)}(x)=\frac{(-1)^{r+1}}{2^{r+1}}+P_{r}(x) e^{2 x}
$$

Combining the latter identity and (9.1), we get

$$
V_{2}^{\left(\sigma_{1}=1\right)}(x)=\frac{(-1)^{r}}{2^{r+1}}+\left(-P_{r}(x)+\frac{x^{r}}{r !}\right) e^{2 x} .
$$

The connecting relations. We now establish relations (2.23) and (2.29). Instead of (3.12), we will use the identity

$$
e^{2 x} S_{k-2}(x)=S_{k}^{\prime \prime}(x)-S_{k}^{\prime}(x)
$$


which can be obtained from (3.6) by a straightforward computation. Combining (9.1) and (9.9), we can write

$$
\left(V_{2}^{\left(\sigma_{1}=1\right)}(x)+V_{2}^{\left(\sigma_{1}=2\right)}(x)\right) S_{k-2}(x)=\frac{x^{r}}{r !}\left(S_{k}^{\prime \prime}(x)-S_{k}^{\prime}(x)\right),
$$

and, after a routine coefficient extraction based on (3.15), we obtain

$$
\left[\frac{x^{n}}{n !}\right]\left(V_{2}^{\left(\sigma_{1}=1\right)}(x)+V_{2}^{\left(\sigma_{1}=2\right)}(x)\right) S_{k-2}(x)=\left(\begin{array}{l}
n \\
r
\end{array}\right)\left(S_{n+2-r, k}-S_{n+1-r, k}\right) .
$$

Summing the both sides of this identity over all integers $k \geq 0$, we obtain

$$
\left[\frac{x^{n}}{n !}\right]\left(V_{2}^{\left(\sigma_{1}=1\right)}(x)+V_{2}^{\left(\sigma_{1}=2\right)}(x)\right) B(x)=\left(\begin{array}{l}
n \\
r
\end{array}\right)\left(B_{n+2-r}-B_{n+1-r}\right) .
$$

It suffices now to divide expression (9.10) by $B_{n}$ and expression (9.11) by $S_{n, k}$, respectively, to obtain the relations (2.23) and (2.29).

Exact average values in the case $\sigma_{1}=2$. Combining (9.7) and (9.9), we can write

$$
V_{2}^{\left(\sigma_{1}=2\right)} S_{k-2}(x)=\frac{(-1)^{r+1}}{2^{r+1}} S_{k-2}(x)+P_{r}(x) S_{k}^{\prime \prime}(x)-P_{r}(x) S_{k}^{\prime}(x) .
$$

After a routine coefficient extraction based on (3.15), we obtain

$$
\begin{gathered}
{\left[\begin{array}{c}
x^{n} \\
n !
\end{array} V_{2}^{\left(\sigma_{1}=2\right)}(x) S_{k-2}(x)=\sum_{j=0}^{r} \frac{(-1)^{r-j}}{j ! 2^{r+1-j}}(n)_{j}\left(S_{n+2-j, k}-S_{n+1-j, k}\right)\right.} \\
+\frac{(-1)^{r+1}}{2^{r+1}} S_{n, k-2} .
\end{gathered}
$$

A rearrangement of the terms of the sum on the right hand side gives

$$
\begin{aligned}
{\left[\frac{x^{n}}{n !}\right] V_{2}^{\left(\sigma_{1}=2\right)}(x) S_{k-2}(x)=} & \frac{(-1)^{r+1}}{2^{r+1}} S_{n, k-2}+\sum_{j=0}^{r} p_{j}(n) S_{n+2-j, k} \\
& -\frac{1}{2}\left(\begin{array}{l}
n \\
r
\end{array}\right) S_{n+1-r, k},
\end{aligned}
$$

where $p_{j}(n)$ is given by $(2.24)$.

Division of expression (9.12) by $S_{n, k}$ gives the exact value of $\mu_{n, k}^{\left(\sigma_{1}=2\right)}$ given in Theorem 2.8, while the exact average value of $\mu_{n}^{\left(\sigma_{1}=2\right)}$ given in Theorem 2.8 can be obtained easily from the exact value of $\mu_{n, k}^{\left(\sigma_{1}=2\right)}$ by using (3.30).

Asymptotic approximations of the average values in the case $\sigma_{1}=2$. The exact average value of $\mu_{n}^{\left(\sigma_{1}=2\right)}$ we found is

$$
\mu_{n}^{\left(\sigma_{1}=2\right)}=\frac{(-1)^{r+1}}{2^{r+1}}+\sum_{j=0}^{r} p_{j}(n) \frac{B_{n+2-j}}{B_{n}}-\frac{1}{2}\left(\begin{array}{c}
n \\
r
\end{array}\right) \frac{B_{n+1-r}}{B_{n}} .
$$


It follows easily from Lemma 3.3 and basic representations of binomial coefficients that, as $n \rightarrow \infty$, we have

$$
\begin{aligned}
p_{j}(n) \frac{B_{n+2-j}}{B_{n}} & =\frac{(-1)^{r-j}}{2^{r+1-j}}\left(\left(\begin{array}{c}
n \\
j
\end{array}\right)+\frac{1}{2}\left(\begin{array}{c}
n \\
j-1
\end{array}\right)\right) \frac{B_{n+2-j}}{B_{n}} \\
& =\frac{(-1)^{r-j}}{2^{r+1-j}} \frac{n^{j}}{j !}\left(1+O\left(\frac{1}{n}\right)\right)\left(\frac{n}{\log n}\right)^{2-j}\left(1+(2-j) \frac{\log \log n}{\log n}(1+o(1))\right) \\
& =\frac{(-1)^{r-j}}{2^{r+1-j}} \frac{1}{j !} n^{2}(\log n)^{j-2}\left(1-(j-2) \frac{\log \log n}{\log n}+o\left(\frac{\log \log n}{\log n}\right)\right)
\end{aligned}
$$

for any integer $j \geq 0$. Similarly, as $n \rightarrow \infty$, one can prove

$$
\left(\begin{array}{l}
n \\
r
\end{array}\right) \frac{B_{n+1-r}}{B_{n}}=\frac{1}{r !} n(\log n)^{r-1}\left(1-(r-1) \frac{\log \log n}{\log n}(1+o(1))\right) .
$$

Combining (9.13) and (9.14), we get after basic comparisons

$$
\mu_{n}^{\left(\sigma_{1}=2\right)}=\frac{1}{2 r !} n^{2}(\log n)^{r-2}\left(1-(r-2) \frac{\log \log n}{\log n}+o\left(\frac{\log \log n}{\log n}\right)\right),
$$

which is exactly $(2.25)$ in the case $\sigma_{1}=2$.

The exact average value of $\mu_{n, k}^{\left(\sigma_{1}=2\right)}$ we found is

$$
\mu_{n, k}^{\left(\sigma_{1}=2\right)}=\sum_{j=0}^{r} p_{j}(n) \frac{S_{n+2-j, k}}{S_{n, k}}-\frac{1}{2}\left(\begin{array}{l}
n \\
r
\end{array}\right) \frac{S_{n+1-r, k}}{S_{n, k}}+\frac{(-1)^{r+1}}{2^{r+1}} \frac{S_{n, k-2}}{S_{n, k}} .
$$

It follows easily from Lemma 3.2 and basic representations of binomial coefficients that, as $n \rightarrow \infty$, we have

$$
\begin{aligned}
\mu_{n, k}^{\left(\sigma_{1}=2\right)}= & \sum_{j=0}^{r} \frac{(-1)^{r-j}}{2^{r+1-j}}\left(\left(\begin{array}{c}
n \\
j
\end{array}\right)+\frac{1}{2}\left(\begin{array}{c}
n \\
j-1
\end{array}\right)\right)\left(k^{2-j}+O\left(\left(1-\frac{1}{k}\right)^{n}\right)\right) \\
& -\frac{1}{2}\left(\begin{array}{c}
n \\
r
\end{array}\right)\left(k^{1-r}+O\left(\left(1-\frac{1}{k}\right)^{n}\right)\right)+\frac{(-1)^{r+1}}{2^{r+1}} O\left(\left(1-\frac{2}{k}\right)^{n}\right) \\
= & \sum_{j=0}^{r} \frac{(-1)^{r-j}}{2^{r+1-j}}\left(\left(\begin{array}{c}
n \\
j
\end{array}\right)+\frac{1}{2}\left(\begin{array}{c}
n \\
j-1
\end{array}\right)\right) k^{2-j}-\frac{1}{2}\left(\begin{array}{c}
n \\
r
\end{array}\right) k^{1-r}+O\left(n^{r}\left(1-\frac{1}{k}\right)^{n}\right),
\end{aligned}
$$

which is a refinement of the asymptotic approximation (2.30) given in Theorem 2.8.

The case $\sigma_{1}=1$. The exact and asymptotic approximations of the average value $\mu_{n}^{\left(\sigma_{1}=1\right)}\left(\mu_{n, k}^{\left(\sigma_{1}=1\right)}\right.$, respectively) given in Theorem 2.7 (Theorem 2.8, respectively) can be easily obtained from the exact and asymptotic approximations of $\mu_{n}^{\left(\sigma_{1}=2\right)}\left(\mu_{n, k}^{\left(\sigma_{1}=2\right)}\right.$, respectively) obtained earlier combined with relation (2.23) (respectively (2.29)). The 
details are left to the reader.

Patterns of length 2. To end the proof of our theorems, we need to establish assertion $(v)$ of Theorem 2.7. In fact, we have already shown in Section 3.3 that (2.26) is

an asymptotic approximation of $\mu_{n}^{(21)}$ (which is actually equal to $\mu_{n}^{\mathrm{inv}}$ as we remarked earlier). Almost the same asymptotic computation shows that (2.26) is an asymptotic approximation of $\mu_{n}^{(12)}$ whose exact value is given in (2.28) (the details are left to the reader).

This concludes the proof of Theorem 2.7 and Theorem 2.8.

\subsection{Remarks}

The notion of pattern containment in set partitions we have considered in this section is due to Sagan [31]. There is also an older and different notion of pattern containment in set partitions due to Klazar (see e.g. [23]). Actually, it can be shown that the statistic 'number of occurrences of a 2-partition' (according to Klazar's definition) is also a Zstatistic. One can show by either applying the methodology developed in this paper or by direct combinatorial arguments that, with Klazar's definition of pattern containment, the average number of occurrences of any 2-partition $\pi$ of $[r]$ in a random set partition of $[n]$ is given by

$$
\left(\begin{array}{l}
n \\
r
\end{array}\right) \frac{B_{n+2-r}-B_{n+1-r}}{B_{n}}
$$

Mansour, Shattuck and Yan [26] have considered a restricted case of pattern containment in set partitions where it is required that the occurrence has to be contiguous. They obtained some average value results for numbers of restricted occurrences of some patterns. Note that their results can not be recovered with the methodology of this paper since, in the contiguous case, numbers of occurrences are not Z-statistics.

\section{Concluding remarks}

\subsection{Average values of Z-statistics of depth $r$}

There is a natural way to generalize Z-statistics. Given an integer $r \geq 1$, we will say that a set partition statistic $S T A T$ is a Z-statistic of depth $r$ if for any $\pi \in \Pi$,

$$
\operatorname{STAT}(\pi)=\sum_{A_{1}, A_{2}, \ldots, A_{r} \in \pi} \operatorname{STAT}\left(\operatorname{st}\left(A_{1} / A_{2} / \cdots / A_{r}\right)\right)
$$

Examples of Z-statistics of depth $r$ which can be found in the literature are the numbers of $r$-crossings and $r$-nestings of arcs [4], the number of occurrences of an $r$-pattern with Sagan's definition (see Section 9.2) and the number of occurrences of an $r$-partition with Klazar's definition (see Section 9.2). 
Theorem 3.1 can be generalized in a straightforward manner to simplify the computation of average values of Z-statistics of depth $r$. Given a Z-statistic of depth $r$ stat, let $v_{n, r}^{\text {stat }}:=\sum_{\pi \in \Pi_{n}^{r}}$ stat $(\pi)$, and let $V_{r}^{\text {stat }}(x)$ be the exponential generating function of the sequence $\left(v_{n, r}^{\text {stat }}\right)_{n \geq 0}$, i.e.,

$$
V_{r}^{\text {stat }}(x)=\sum_{n \geq 0} v_{n, r}^{\text {stat }} \frac{x^{n}}{n !}=\sum_{n \geq 0} \sum_{\pi \in \Pi_{n}^{r}} \operatorname{stat}(\pi) \frac{x^{n}}{n !} .
$$

Then one can prove the following result.

Theorem 10.1. Let stat be a Z-statistic of depth $r$. Denote by $\mu_{n}$ (respectively $\mu_{n, k}$ ) the average value of the statistic stat in a random set partition of $[n]$ (respectively of $[n]$ into $k$ blocks). Then we have:

$$
\begin{aligned}
\mu_{n} & =\frac{1}{B_{n}}\left[\frac{x^{n}}{n !}\right] V_{r}^{\text {stat }}(x) B(x) \\
\mu_{n, k} & =\frac{1}{S_{n, k}}\left[\frac{x^{n}}{n !}\right] V_{r}^{\text {stat }}(x) S_{k-2}(x) .
\end{aligned}
$$

Note that the point of the above result is that the computation of average values of a Z-statistic of depth $r$ in a random set partition is essentially equivalent to the computation of its average value in a random $r$-partition.

As open problems, we ask the following questions. What are the (exact and asymptotic) average values of the numbers of $r$-crossings, $r$-nestings, occurrences of an $r$-pattern in a random set partition of $[n]$ ?

\subsection{Other exponential families}

In this paper we concentrated on set partitions but we might consider any other exponential family. An exponential family is, roughly speaking, a set of structures that are built out of connected pieces (see e.g. [36] for a precise definition). One can easily establish a generalized version of Theorem 3.1 for exponential families to simplify computation of average values. We do not think that it is necessary to state the generalization, but we give a typical illustration of application.

Consider a natural generalization of matchings, namely the (exponential family of) set partitions all blocks of which are of size $m$ ( $m$ is a given integer $\geq 1$ ). For these partitions, we can consider the statistics 'numbers of crossings in linear and circular representations' and it is easy to see that these statistics are still Z-statistics on such partitions. Then one can prove the following results.

- The average number $\mu_{\left(m^{k}\right)}^{(\ell)}$ of linear crossings in a random set partition into $k$ blocks of size $m, m \geq 1$, is given by

$$
\mu_{\left(m^{k}\right)}=\left(\begin{array}{l}
k \\
2
\end{array}\right)\left(m-2+\frac{2}{\left(\begin{array}{c}
2 m \\
m
\end{array}\right)}\right) .
$$


- The average number $\mu_{\left(m^{k}\right)}^{(c)}$ of circular crossings in a random set partition into $k$ blocks of size $m, m \geq 3$, is given by

$$
\mu_{\left(m^{k}\right)}=\left(\begin{array}{l}
k \\
2
\end{array}\right)\left(m+\frac{1}{2}+\frac{1}{2(2 m-1)}-\frac{4 m}{\left(\begin{array}{c}
2 m \\
m
\end{array}\right)}\right) .
$$

Note that, when $m=2$, we recover already known results (see e.g. [11, 28]).

Acknowledgments. The author would like to thank Christian Krattenthaler and Einar Steingrímsson for their encouragement during the preparation of this paper, and the anonymous referee for his helpful suggestions concerning the presentation of this paper. Special acknowledgement is given to Christian Krattenthaler for a careful proofreading of this paper. All remaining errors are the author's.

\section{References}

[1] P. Biane, Some properties of crossings and partitions, Discrete Math. 175 (1997), $41-53$.

[2] D. Bressoud and D. Zeilberger, A proof of Andrews' $q$-Dyson conjecture, Discrete Math. 54 (1985), no. 2, 201-224.

[3] L. Carlitz, q-Bernoulli numbers and polynomials, Duke Math. J. 15 (1948), 987-1000.

[4] W.Y.C. Chen, E.Y.P. Deng, R.R.X. Du, R.P. Stanley and C.H. Yan, Crossings and nestings of matchings and partitions, Trans. Amer. Math. Soc. 359 (2007), no. 4, $1555-1575$.

[5] W.Y.C. Chen, I. Gessel, C.H. Yan and A.L.B. Yang, A major index for matchings and set partitions, J. Combin. Theory Ser. A 115 (2008), no. 6, 1069-1076.

[6] D. Drake and J.S. Kim, $k$-Distant crossings and nestings of matchings and partitions, Discrete Math. Theoret. Comput. Sci. Proc. AK, 349-360, (2009).

[7] A. de Médicis and P. Leroux, A unified combinatorial approach for $q^{-}$(and $p, q-$ ) Stirling numbers, J. Statist. Plann. Inference 34 (1993), no. 1, 89-105.

[8] R.S. Deodhar and M.K. Srinivasan, An inversion number statistic on set partitions, Electron. Notes Discrete Math. 15 (2003), 84-86 (electronic).

[9] R. Ehrenborg and M. Readdy, Juggling and applications to $q$-analogues, Discrete Math. 157 (1996), no. 1-3, 107-125.

[10] P. Flajolet, Combinatorial aspects of continued fractions, Discrete Math. 41 (1982), $145-153$.

[11] P. Flajolet and M. Noy, Analytic combinatorics of chord diagrams, Formal power series and algebraic combinatorics (Moscow, 2000), 191-201, Springer, Berlin, 2000.

[12] P. Flajolet and R. Schott, Non-overlapping partitions, continued fractions, Bessel functions and a divergent series, European J. Combin. 11 (1990), 412-432. 
[13] P. Flajolet, R. Sedgewick, Analytic combinatorics. Cambridge University Press, Cambridge, 2009.

[14] J. Galovich and D. White, Mahonian $Z$ statistics, Discrete Math. 307 (2007), no. 19-20, 2341-2350.

[15] A.M. Garsia and J. B. Remmel, $Q$-counting rook configurations and a formula of Frobenius, J. Combin. Theory Ser. A 41 (1986), 246-275.

[16] H. W. Gould, The $q$-Stirling numbers of the first and second kinds, Duke Math. J. 28 (1961), 281-289.

[17] W.P. Johnson, Some applications of the $q$-exponential formula, Proceedings of the 6th Conference on Formal Power Series and Algebraic Combinatorics (New Brunswick, NJ, 1994), Discrete Math. 157 (1996), no. 1-3, 207-225.

[18] M. Josuat-Vergès and M. Rubey, Crossings, Motzkin paths and moments, Discrete Math. 311 (2011), Issues 18-19, 2064-2078.

[19] A. Kasraoui, On the limiting distribution of some numbers of crossings in set partitions, in preparation.

[20] A. Kasraoui, Études combinatoires sur les permutations et partitions d'ensemble, Ph.D. thesis, Université Lyon 1, 2009.

[21] A. Kasraoui, D. Stanton and J. Zeng, The combinatorics of Al-Salam-Chihara qLaguerre polynomials, Advances in Applied Mathematics 47 (2011), Issue 2, 216-239.

[22] A. Kasraoui and J. Zeng, Distribution of crossings, nestings and alignments of two edges in matchings and partitions, Electron. J. Combin. 13 (2006), no. 1, Research Paper 33.

[23] M. Klazar, Counting pattern-free set partitions. I. A generalization of Stirling numbers of the second kind. European J. Combin. 21 (2000), no. 3, 367-378.

[24] G. Ksavrelof and J. Zeng, Nouvelles statistiques de partitions pour les $q$-nombres de Stirling de seconde espèce, Discrete Math. 256 (2002), Issue 3, 743-758.

[25] P. Leroux, Reduced matrices and $q$-log-concavity properties of $q$-Stirling numbers, J. Combin. Theory Ser. A 54 (1990), no. 1, 64-84.

[26] T. Mansour, M. Shattuck and S.H.F. Yan, Counting subwords in a partition of a set, Electron. J. Combin. 17 (2010), no. 1, Research Paper 19, 21 pp.

[27] S. Milne, Restricted growth functions, rank row matching of partition lattices, and q-Stirling numbers, Adv. Math. 43 (1982), 173-196.

[28] J. Riordan, The distribution of crossings chords joining pairs of $2 n$ points on a circle, Math. Computation 29 (1975), 215-222.

[29] V.N. Sachkov, Probabilistic methods in combinatorial analysis. Translated from the Russian. Revised by the author. Encyclopedia of Mathematics and its Applications, 56. Cambridge University Press, Cambridge, 1997.

[30] B. Sagan, A maj statistic for set partitions, European J. Combin. 12 (1991), 69-79.

[31] B. Sagan, Pattern Avoidance in Set Partitions, Ars Combin. 94 (2010), 79-96. 
[32] B. Salvy and J. Shackell, Symbolic asymptotics: functions of two variables, implicit functions, J. Symbolic Comput. 25 (1998), no. 3, 329-349.

[33] R. Simion, Non crossing partitions, Discrete Math. 217 (2000), 367-409.

[34] M. Wachs and D. White, $p, q$-Stirling numbers and set partition statistics, J. Combin. Theory Ser. A 56 (1991), 27-46.

[35] D. White, Interpolating Set Partition Statistics, J. Combin. Theory Ser. A 68 (1994), $262-295$.

[36] H. Wilf, generatingfunctionology, Academic Press, Inc., Boston, MA, 1990. 